DRAFT VERSION JANUARY 11, 2021

Preprint typeset using $\mathrm{LATEX}_{\mathrm{E}}$ style emulateapj v. 5/2/11

\title{
SEARCH FOR BRIGHT NEARBY M DWARFS WITH VIRTUAL OBSERVATORY TOOLS
}

\author{
M. Aberasturi, J. A. Caballero, B. Montesinos, M. C. Gálvez-Ortiz, E. Solano and E. L. Martín \\ Centro de Astrobiología (CSIC-INTA), Departamento de Astrofísica, PO Box 78, E-28691 Villanueva de la Cañada, Madrid, Spain \\ Draft version January 11, 2021
}

\section{ABSTRACT}

Using Virtual Observatory tools, we cross-matched the Carlsberg Meridian 14 and the 2MASS Point Source catalogs to select candidate nearby bright $\mathrm{M}$ dwarfs distributed over $\sim 25,000 \mathrm{deg}^{2}$. Here, we present reconnaissance low-resolution optical spectra for 27 candidates that were observed with the Intermediate Dispersion Spectrograph at the $2.5 \mathrm{~m}$ Isaac Newton Telescope $(\mathcal{R} \approx 1600)$. We derived spectral types from a new spectral index, $\Re$, which measures the ratio of fluxes at $7485-7015 \AA$ and 7120-7150^. We also used VOSA, a Virtual Observatory tool for spectral energy distribution fitting, to derive effective temperatures and surface gravities for each candidate. The resulting 27 targets were M dwarfs brighter than $J=10.5 \mathrm{mag}, 16$ of which were completely new in the Northern hemisphere and 7 of which were located at less than $15 \mathrm{pc}$. For all of them, we also measured $\mathrm{H} \alpha$ and $\mathrm{Na}$ I pseudo-equivalent widths, determined photometric distances, and identified the most active stars. The targets with the weakest sodium absorption, namely J0422+2439 (with X-ray and strong H $\alpha$ emissions), J0435+2523, and J0439+2333, are new members in the young Taurus-Auriga star-forming region based on proper motion, spatial distribution, and location in the color-magnitude diagram, which reopens the discussion on the deficit of M2-4 Taurus stars. Finally, based on proper motion diagrams, we report on a new wide M-dwarf binary system in the field, LSPM J0326+3929EW.

Subject headings: astronomical databases: miscellaneous - open clusters and associations: individual (Taurus-Auriga) - stars: late-type - stars: low-mass - stars: pre-main sequence virtual observatory tools

\section{INTRODUCTION}

$\mathrm{M}$ dwarfs are the most common stars in the universe. Not only is the closest star to the Sun an M dwarf (Proxima Centauri), but also $66 \%$ of the nearest stars in our Galactic neighborhood $(d<10 \mathrm{pc})$

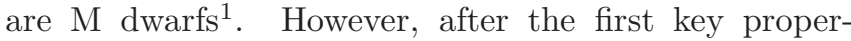
motion surveys in the first decades of the 20th century (van Maanen 1915; Wolf 1919; Ross 1939), the famous catalogs of Gliese (1969), Giclas et al. (1971, 1978), Luyten (1979a,b), Gliese \& Jahreiss (1991), and the concluding spectroscopic studies of Kirkpatrick et al. (1991) and the RECONS Research Consortium of Nearby Stars (Reid et al. 1995; Hawley et al. 1997) at the end of the millennium, $\mathrm{M}$ dwarfs were relatively forgotten in the first decade of the current century. This apparent falling into oblivion, apart from a few honorable exceptions (e.g., Lépine et al. 2003b; Lépine \& Shara 2005), was mostly due to many stellar astronomers focusing on the search and characterization of cooler objects with later spectral types: L, T, and, quite recently, Y (e.g., Martín et al. 1997, 1999, Kirkpatrick et al. 1999; Burgasser et al. 2002; Cushing et al. 2011).

Almost twenty years later, there have been a rebirth of M-dwarf studies. A few examples in the last four years are the possible existence of exoplanets in habitable zones around M dwarfs (Charbonneau et al. 2009; Anglada-Escudé et al. 2012; Bonfils et al. 2013), a high occurrence of Earth-like-radius transiting exoplanets around cool stars in the Kepler field (Batalha et al. 2010; Howard et al. 2012; Muirhead et al. 2012) and close to the Sun (Apps et al. 2010; Johnson et al.

\footnotetext{
1 http://www.recons.org/
}

2010; Bonfils et al. 2011), transmission spectra of superearths around M dwarfs (Miller-Ricci \& Fortney 2010; Berta et al. 2012), the luminosity and mass functions of low-mass stars in the solar neighborhood from Sloan data (Bochanski et al. 2010; West et al. 2011), low contrast ratios that favor the detection of very faint, close-in (planetary) companions Chauvin et al. 2010), high-precision dynamical masses of very lowmass binaries (Konopacky et al. 2010), the complete new field of M-dwarf metallicity (Schlaufman \& Laughlin 2010; Roias-Avala et al. 2010, 2012), fragile low-mass binaries (Burningham et al. 2010; Faherty et al. 2010; Dhital et al. 2010), or even a rebith of activity studies in light of new magnetohydrodynamic models (Morin et al. 2010; Browning et al. 2010). Many exoplanet hunters turn now their eyes to $M$ dwarfs, both with current instruments (CRIRES: Bean et al. 2010; NIRSPEC: Blake et al. 2010; MEarth: Irwin et al. 2011, Berta et al. 2013; APOGEE: Zasowski et al. 2013; $\mathrm{Ke}$ pler: Martín et al.|2013) and with future ones (SPIRoU: Artigau et al. 2011; CARMENES: Quirrenbach et al. 2012; HPF: Mahadevan et al. 2012; TESS: Ricker et al. 2010; EChO: Tinetti et al. 2012). In parallel, many research teams now focus on searching for the best M dwarfs for radial-velocity and transit exoplanet surveys (e.g., Reiners et al. 2010; Lépine \& Gaidos 2011; Lépine et al. 2013; Frith et al. 2013; Caballero et al. 2013), apart from characterizing in detail such potential targets.

In this work we search for unidentified bright intermediate $\mathrm{M}$ dwarfs in the solar neighborhood using Virtual Observatory ( $\mathrm{VO}^{2}$ ) techniques. The VO

\footnotetext{
2 http://www.ivoa.net
} 

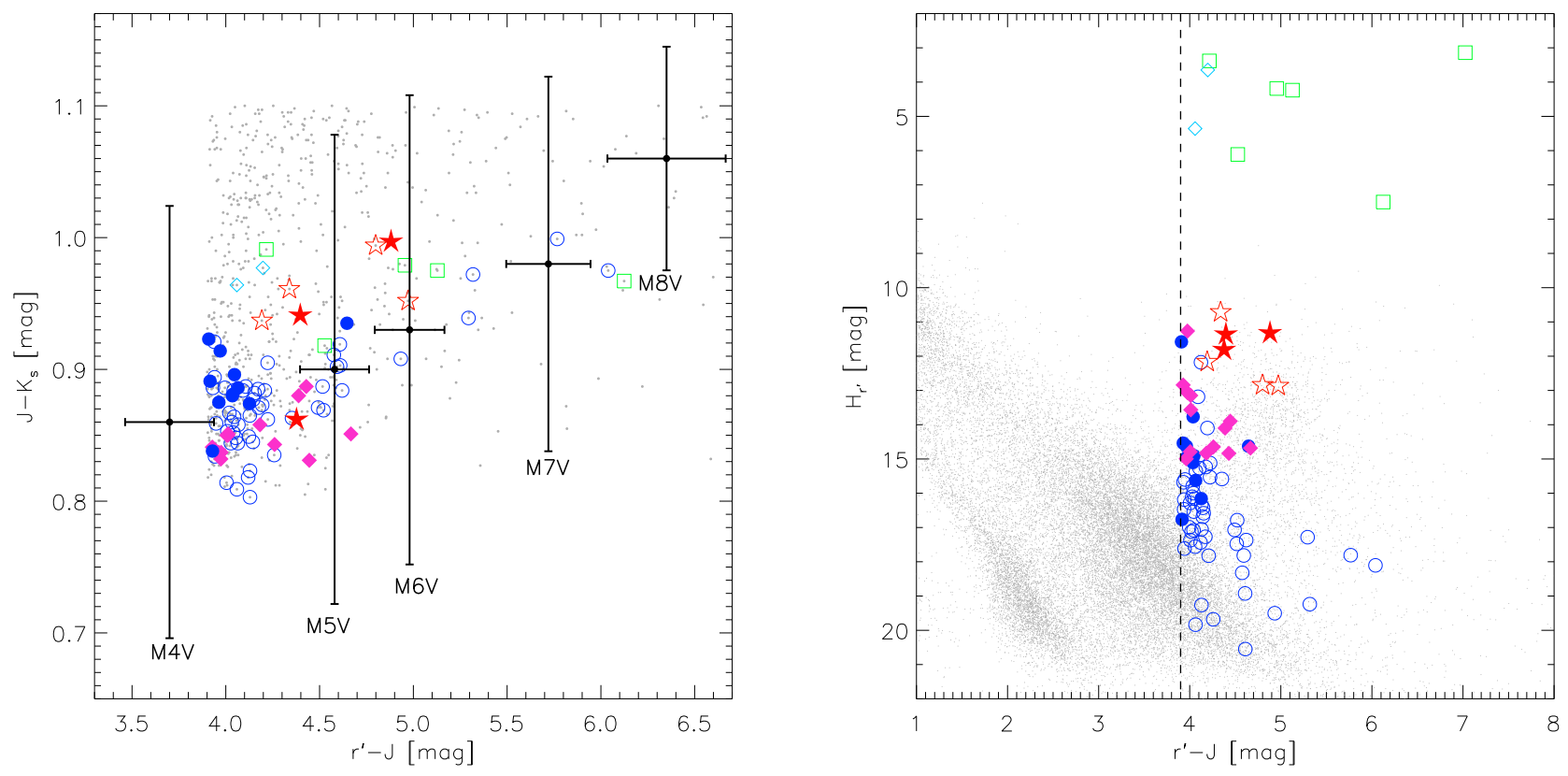

FIG. 1. - Left panel: $J-K_{s}$ vs. $r^{\prime}-J$ color-color diagram. Small gray solid points represent the full cross-matched sources. Filled symbols are for stars spectroscopically investigated in this work, while open symbols are for the cross-matched known stars in Table 1 New field M dwarfs reported here for the first time are drawn with (magenta) rhombs, previously known field M dwarfs with (blue) circles, members in Taurus-Auriga with (red) stars, M giants with (green) squares, and reddened Cygnus OB2 massive stars with (cyan) rhombs. Black error bars represent the average colors of typical dwarfs of spectral types M4 V to M8 V (West et al. 2008). Right panel: same as left panel but the tiny gray points represent stars in Lépine \& Shara (2005) with the CMC14 counterpart. The vertical dashed line at $r^{\prime}-J$ $=3.9 \mathrm{mag}$ indicates our color cut. The two Galactic disk dwarf and halo subdwarf sequences are distinguishable in the bottom left corner. is "an international initiative designed to provide the astronomical community with the data access and the research tools necessary to enable the exploration of the digital, multi-wavelength universe that is resident in the astronomical data archives". The VO is already an operational research infrastructure as demonstrated by the growing number of papers using VO tools (see, for instance, Caballero 2009; Valdivielso et al. 2009; Aberasturi et al. 2011; Lodieu et al.|2012; Luhman 2013; López Martí et al. [2013; Malo et al. [2013, or Stelzer et al. 2013 for recent examples of VO science papers focused on low-mass stars). Besides, in this work we combine our VO search with a low-resolution spectroscopic follow-up, an astrometric and photometric study, and an activity analysis (based on our $\mathrm{H} \alpha$ measurements and X-ray emission from public databases) to successfully identify not only potential targets for exoplanet hunting at less than $20 \mathrm{pc}$, but also to serendipitously identify three young very low-mass stars in the TaurusAuriga region.

\section{OBSERVATIONS AND ANALYSIS}

\subsection{Candidate Selection}

TABLE 1 The 97 cross-matched objects that passed the final filters and were not spectroscopically analyzed

\begin{tabular}{|c|c|c|c|c|}
\hline Name & $\begin{array}{l}\alpha(\mathrm{J} 2000) \\
\text { hh mm ss }\end{array}$ & $\begin{array}{l}\delta(\mathrm{J} 2000) \\
\mathrm{dd} \mathrm{mm} \text { ss }\end{array}$ & SpT & Class \\
\hline LP $404-33$ & 000853.92 & +205025.2 & $\mathrm{M} 4.5$ & Dwarf \\
\hline TYC 2268-648-1 & 001322.97 & $+33: 47: 02.2$ & & Giant? \\
\hline GJ 1011 & 002328.03 & +241824.4 & M4.0 & Dwarf \\
\hline G $130-68$ & 002434.78 & +300229.5 & M4.5 & Dwarf \\
\hline $\mathrm{G} 132-25 \mathrm{AB}$ & 004556.63 & +334711.0 & $\mathrm{M} 4.5+$ & Dwarf \\
\hline G $69-32$ & 005448.03 & +273103.6 & M4.5 & Dwarf \\
\hline IX And & 010140.56 & +375346.5 & M4.0: & Giant \\
\hline LSR J0155+3758 & 015502.30 & $\begin{array}{r}+375802.8 \\
\end{array}$ & M5.0 & Dwarf \\
\hline 2MASS J02132062+3648506 & 021320.63 & +364850.7 & M4.5 & Dwarf \\
\hline LP $245-10$ & 021709.93 & +352633.0 & M5.0 & Dwarf \\
\hline FBS L 14-14 & 023541.64 & +260303.2 & M6.5: & Giant \\
\hline G $36-26$ & 023644.13 & +224026.5 & M5.0 & Dwarf \\
\hline TYC 1779-1379-1 & 023631.24 & $\begin{array}{r}+293555.7 \\
\end{array}$ & & Giant? \\
\hline LSPM J0256+2359 & 025613.96 & +235910.5 & M5.5 & Dwarf \\
\hline LP $355-27$ & 030746.82 & +245755.6 & M4.5 & Dwarf \\
\hline G $6-7$ & 032644.96 & +191440.3 & M4.5 & Dwarf \\
\hline RX J0332.6+2843 & 033235.79 & +284355.5 & M4.0 & Dwarf \\
\hline XEST 16-045 & 042039.18 & $\begin{array}{r}271731.7 \\
+\end{array}$ & M4.0: & Young \\
\hline GJ 1070 & 042233.49 & +390043.7 & M5.0 & Dwarf \\
\hline G $8-31$ & 042259.26 & +255914.8 & M4.0 & Dwarf \\
\hline FW Tau AB & 042929.71 & +261653.2 & $\mathrm{M} 5.5 \mathrm{e}+$ & Young \\
\hline V546 Per & 043025.27 & +395100.0 & M4.5 & Dwarf \\
\hline
\end{tabular}


TABLE 1. Continued.

\begin{tabular}{|c|c|c|c|c|}
\hline Name & $\alpha(\mathrm{J} 2000)$ & $\delta(\mathrm{J} 2000)$ & SpT & Class \\
\hline$\nabla 927$ Tau AB & 043123.82 & +241052.9 & $\mathrm{M} 4.5 \mathrm{e}+$ & Young \\
\hline G $8-41$ & 043333.93 & +204446.2 & M4.0 & Dwarf \\
\hline LP $415-1644$ & 043721.91 & +192117.4 & 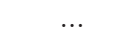 & Dwarf? \\
\hline Haro $6-36$ & 044320.23 & +294006.0 & M5.0e & Young \\
\hline RX J0447.2+2038 & 044712.25 & +203810.9 & M4.5 & Dwarf \\
\hline GJ 1072 & 045050.83 & +220722.5 & M5.0 & Dwarf \\
\hline LSPM J0501+2237 & 050118.03 & +223701.6 & M4.5 & Dwarf \\
\hline HD $285190 \mathrm{BC}$ & 050305.63 & +212236.2 & $\mathrm{M} 4.5+$ & Dwarf \\
\hline IRAS $05090+2027$ & 051203.98 & +203053.7 & $\ldots$ & Giant? \\
\hline [LH98] 190 & 052425.72 & +192207.0 & & Dwarf? \\
\hline V780 Tau A & 054025.71 & +244809.0 & M5.5 & Dwarf \\
\hline LHS 6097 & 055853.33 & +212101.1 & M4.5 & Dwarf \\
\hline G 98-52 A & 061155.99 & +332550.6 & M3.5 & Dwarf \\
\hline $0628+2052$ & 062835.97 & +205237.6 & $\ldots$ & Giant? \\
\hline IRAS $06386+2330$ & 064141.14 & +232741.4 & $\ldots$ & Giant? \\
\hline IRAS $06562+2229$ & 065918.80 & +222453.4 & & Giant? \\
\hline GJ 1093 & 065928.69 & +192057.7 & M5.0 & Dwarf \\
\hline GJ 1096 & 071618.02 & +330910.4 & M4.0 & Dwarf \\
\hline $\mathrm{BD}+202091$ & 082824.90 & +193544.5 & M6.0: & Giant \\
\hline DX Cnc & 082949.34 & +264633.7 & M6.0 & Dwarf \\
\hline $\mathrm{CV}$ Cnc BC & 083137.44 & +192349.5 & $\mathrm{M} 4.0+$ & Dwarf \\
\hline$\rho$ Cnc B & 085240.85 & +281858.9 & M4.0 & Dwarf \\
\hline EI Cnc AB & 085815.19 & +194547.1 & $\mathrm{M} 5.5+$ & Dwarf \\
\hline LP $368-128$ & 090023.59 & +215005.4 & M6.5 & Dwarf \\
\hline 2MASS J09165078+2448559 & 091650.78 & +244856.0 & M4.5 & Dwarf \\
\hline DX Leo B & 093248.27 & +265944.3 & M5.5 & Dwarf \\
\hline Ross 92 & 094102.00 & +220129.2 & M4.5 & Dwarf \\
\hline LHS 2206 & 095355.23 & +205646.0 & M4.5 & Dwarf \\
\hline $\mathrm{BD}+252207$ & 100927.78 & +245731.8 & M5.0: & Giant \\
\hline TYC 1968-490-1 & 101352.77 & +225648.5 & & Giant? \\
\hline G $54-19$ & 101453.16 & +212346.4 & M4.5 & Dwarf \\
\hline LP 374-39 & 112308.00 & +255337.0 & M5.0 & Dwarf \\
\hline G $121-28$ & 115257.91 & +242845.4 & M4.5 & Dwarf \\
\hline G $148-47$ B & 122126.69 & +303837.0 & 20.0 & Dwarf? \\
\hline Sand 58 A & 122127.05 & +303835.7 & M5.0 & Dwarf \\
\hline HD $108421 \mathrm{C}$ & 122657.37 & +270053.7 & M4.5 & Dwarf \\
\hline G $123-45$ & 123628.70 & +351200.8 & M3.5 & Dwarf \\
\hline LP $377-36$ & 123943.54 & +253045.7 & M4.5 & Dwarf \\
\hline Sand 214 & 130650.25 & +305054.9 & M5.0 & Dwarf \\
\hline GJ 1167 A & 130934.95 & +285906.6 & M4.0 & Dwarf \\
\hline EK CVn & 131432.49 & +342055.9 & M6.0: & Giant \\
\hline GJ 1171 & 133031.06 & +190934.0 & M4.5 & Dwarf \\
\hline LP 323-169 & 133239.09 & +305906.6 & M4.5 & Dwarf \\
\hline GJ 1179 A & 134813.41 & +233648.6 & M5.5 & Dwarf \\
\hline G 166-33 & 142959.56 & +293402.9 & M4.0 & Dwarf \\
\hline NLTT 39916 & 151921.23 & +340342.8 & & Dwarf? \\
\hline G $167-47$ & 153154.27 & +285109.6 & M4.5 & Dwarf \\
\hline G $180-11$ AB & 155531.78 & +351202.9 & $\mathrm{M} 4.5+$ & Dwarf \\
\hline HD 190360 B & 200326.52 & +295200.0 & M4.5 & Dwarf \\
\hline $\mathrm{J} 2006+3651$ & 200654.00 & +365148.3 & $\ldots$ & Giant? \\
\hline $\mathrm{J} 2011+3423$ & 201156.42 & +342358.1 & $\ldots$ & Giant? \\
\hline $\mathrm{J} 2014+3943$ & 201455.51 & +394326.7 & $\cdots$ & Dwarf? \\
\hline HD $346301 \mathrm{~A}$ & 201902.83 & +220521.4 & $\ldots$ & Giant? \\
\hline $\mathrm{J} 2022+4030$ & 202214.67 & +403002.7 & $\ldots$ & Giant? \\
\hline $\mathrm{J} 2023+2451$ & 202334.27 & +245120.1 & $\ldots$ & Giant? \\
\hline $\mathrm{J} 2024+3930$ & 202438.71 & +393030.1 & $\ldots$ & Giant? \\
\hline $\mathrm{J} 2026+3733$ & 202616.44 & +373301.2 & ... & Giant? \\
\hline G 210-20 & 202822.08 & +341208.7 & ... & Dwarf? \\
\hline [CPR2002] A25 & 203238.44 & +404044.5 & O8 III & Young \\
\hline $\mathrm{J} 2032+4042$ & 203243.88 & +404217.1 & $\ldots$ & Giant? \\
\hline [CPR2002] A20 & 203302.92 & +404725.4 & $\mathrm{O} 8 \mathrm{II}((\mathrm{f}))$ & Young \\
\hline [CPR2002] A22 & 203311.29 & +404233.7 & $\ldots$ & Giant? \\
\hline G $210-26$ & 203315.77 & +282344.0 & $\ldots$ & Dwarf? \\
\hline $\mathrm{J} 2038+4021$ & 203817.13 & +402106.0 & $\ldots$ & Giant? \\
\hline LSPM J2045+3508 & 204522.16 & +350815.1 & & Dwarf? \\
\hline G 211-9 & 210246.06 & +345436.0 & M4.5 & Dwarf \\
\hline IRAS $21044+2818$ & 210635.76 & +283106.1 & . & Giant? \\
\hline V445 Vul & 210801.33 & +234344.6 & M7.0: & Giant \\
\hline TYC 2710-1557-1 & 210913.22 & +340848.0 & $\ldots$ & Giant? \\
\hline LSR J2124+4003 & 212432.34 & +400400.0 & M6.5 & Dwarf \\
\hline $\mathrm{BD}+294448$ & 213357.94 & +294911.4 & $\ldots$ & Giant? \\
\hline $\mathrm{J} 2154+1914$ & 215451.21 & +191410.7 & $\ldots$ & Giant? \\
\hline G $130-31$ & 235919.80 & +324123.7 & $\ldots$ & Dwarf? \\
\hline G $127-50$ & 224323.13 & +220817.9 & M4.5 & Dwarf \\
\hline GJ 1288 & 234252.74 & +304921.9 & M4.5 & Dwarf \\
\hline
\end{tabular}


TABLE 1. Continued.

\begin{tabular}{|c|c|c|c|c|}
\hline Name & $\alpha(\mathrm{J} 2000)$ & $\delta(\mathrm{J} 2000)$ & $\mathrm{SpT}$ & Class \\
\hline
\end{tabular}

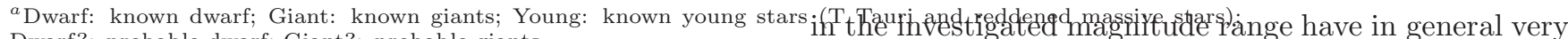
Dwarf?: probable dwarf; Giant?: probable giants

First of all, we cross-matched the whole Carlsberg Meridian Catalogue 14 (CMC143: Copenhagen University et al. 2006 - see Evans et al. 2002 for a description of a previous release) with the Two-Micron All Sky Survey (2MASS; Skrutskie et al. 2006). This correlation was performed with the help of the Aladin sky atlas (Bonnarel et al. 2000) and the Starlink Tables Infrastructure Library Tool Set (STILTS; Taylor 2006). To avoid memory overflow problems, we divided the $25078 \mathrm{deg}^{2}$ of common CMC14-2MASS sky (i.e., the CMC14 area - over $60 \%$ of the whole sphere) into overlapping circular regions of 30 arcmin radius. We used a matching radius of 5 arcsec, which ensured that objects with high proper motions of up to $\mu \sim$ 1700 mas a $^{-1}$ (given the typical baseline between 2MASS and CMC14 astrometric epochs) were not left out. Only the closest 2MASS counterpart to each CMC14 source was considered.

Constraints based on the colors expected for M dwarfs with spectral types M4 V and later were imposed. In particular, we selected objects with colors $r^{\prime}-J>3.9 \mathrm{mag}$ and $0.8 \mathrm{mag}<J-K_{\mathrm{s}}<1.1 \mathrm{mag}$ (e.g., West et al. 2008) and high quality 2MASS flags (AAA).

Since we were interested in bright $\mathrm{M}$ dwarfs, our last restriction was $J<10.5 \mathrm{mag}$. We did not expect to identify any dwarfs later than M8 V $\left(r^{\prime}-J \gtrsim 6.5 \mathrm{mag}\right)$ because the CMC14 completeness magnitude is $r^{\prime} \approx 17.0 \mathrm{mag}$ in the Sloan passband (slightly variable by $0.1-0.2 \mathrm{mag}$ from one sky region to another). After these color and magnitude cuts, we expect to find M4 V (M8 V) stars within $45 \mathrm{pc}(7.5 \mathrm{pc})$ the Sun using the $M_{J}$-SpT relation in Caballero et al. (2008, Table 3). The selection of the resulting 828 sources with $r^{\prime} J H K_{\mathrm{s}}$ photometry is illustrated in the left panel of Figure. 1 .

We prepared a list of high-priority targets for a spectroscopic run planned for the Canarian winter (see Section 2.2). Of the 828 stars, we selected objects visible during the season (i.e., with right ascensions $22 \mathrm{~h}<\alpha<$ $16 \mathrm{~h})$ and with minimum zenith distances during culmination (i.e., with declinations $+18 \operatorname{deg}<\delta<+41 \mathrm{deg}$ ). We imposed an extra cut at colors $J-K_{\mathrm{s}}<1.0 \mathrm{mag}$, thus making the near-infrared color constraint actually 0.8 $\operatorname{mag}<J-K_{\mathrm{s}}<1.0 \mathrm{mag}$. The 125 sources passing these filters were inspected visually with Aladin and classified into five groups: (1) known dwarfs, (2) known giants, (3) known young stars (T Tauri and reddened massive stars), 4) probable giants, and (5) probable dwarfs. There was only one artifact from an incorrect CMC14-2MASS crossmatch of a visual binary, which resulted in a final list of 124 stars. For the classification, we used additional information gathered from the SIMBAD 4 and $\mathrm{AD} \$ 5$ services, and the PPMXL catalog of positions and proper motions on the ICRS (PPMXL; Roeser et al. 2010) and IRAS point source (Helou \& Walker 1988). Field giants

\footnotetext{
3 http://www . ast. cam.ac.uk/ dwe/SRF/cmc14.html

4 http://simbad.u-strasbg.fr/simbad/sim-fid

5 http://adswww.harvard.edu/
} low proper motions, lower than 5 mas a ${ }^{-1}$, near-infrared colors close to the upper limit $\left(J-K_{\mathrm{s}} \approx 1.0 \mathrm{mag}\right)$, flux excess in the IRAS passbands, and, in some cases, photometric variability due to pulsations (see, e.g., the recent VO-based survey for bright Tycho-2 stars with red colors by Jiménez-Esteban et al. 2012).

The 97 cross-matched known dwarfs, giants and young stars with spectral-type determination and probable dwarfs and giants without spectral typing are shown in Table 1. All the known dwarfs (55) except one have spectral types M4.0 V or later (the exception is G 98$52 \mathrm{~A}$, which has an M3.5 V spectral type). Table 1 includes four pre-main sequence $\mathrm{T}$ Tauri stars in TaurusAuriga (XEST 16-045, FW Tau AB, V927 Tau AB, and Haro 6-36; see Section (3.4) and a couple of reddened massive stars in Cygnus OB2 ([CPR2002] A20 and [CPR2002] A25; Comerón et al. 2002). Field M giants, T Tauri stars, and reddened Cygnus OB2 massive stars fall in well-defined locations in a reduced-proper-motions diagram, as the one shown in the right panel of Figure. 1

The remaining 27 high-priority dwarf candidates, shown in Table 2 were selected for spectroscopic follow-up. They all had proper motions greater than 20 mas a $^{-1}$, no IRAS detection, and magnitudes and colors consistent with intermediate or late M spectral type and luminosity class V. Of them, only 11 were previously identified and classified as M dwarf candidates based only on photometry by Giclas et al. (1959, 1961), McCarthy \& Treanor (1964), Luvten (1979b), Fleming (1998), Lépine \& Shara (2005), and Lépine \& Gaidos (2011).

\subsection{Spectroscopy}

On 2012 January 11-13, we used the Intermediate Dispersion Spectrograph (IDS) at the $2.5 \mathrm{~m}$ Isaac Newton Telescope (INT) in the Observatorio del Roque de Los Muchachos (La Palma, Spain). We used the configuration with the Red +2 detector, the R300V grating centered on $550 \mathrm{~nm}$, and the 1.0 arcsec wide slit, which provided a resolution $\mathcal{R} \approx 1600$ over a wide wavelength interval from 360 to $900 \mathrm{~nm}$. The actual useful wavelength interval was, however, a bit narrower, from 425 to $825 \mathrm{~nm}$.

We collected low-resolution spectra of the 27 highpriority dwarf candidates and 5 reference stars for spectral-type determination, which are shown in Table 3 . Exposure times ranged between 700 and 2400 s, depending on target brightness. Raw data were next reduced following standard procedures within the IRAF environment (bias and flat-field correction, cosmic-ray rejection, and optimal extraction). Wavelength calibration was carried out with spectra of $\mathrm{Cu}-\mathrm{Ne}$ arc lamps taken during the run. Figure. 2 shows the 32 final spectra of bright M dwarf candidates and reference stars sorted by spectral type.

\section{RESULTS}

\subsection{Spectral Types}



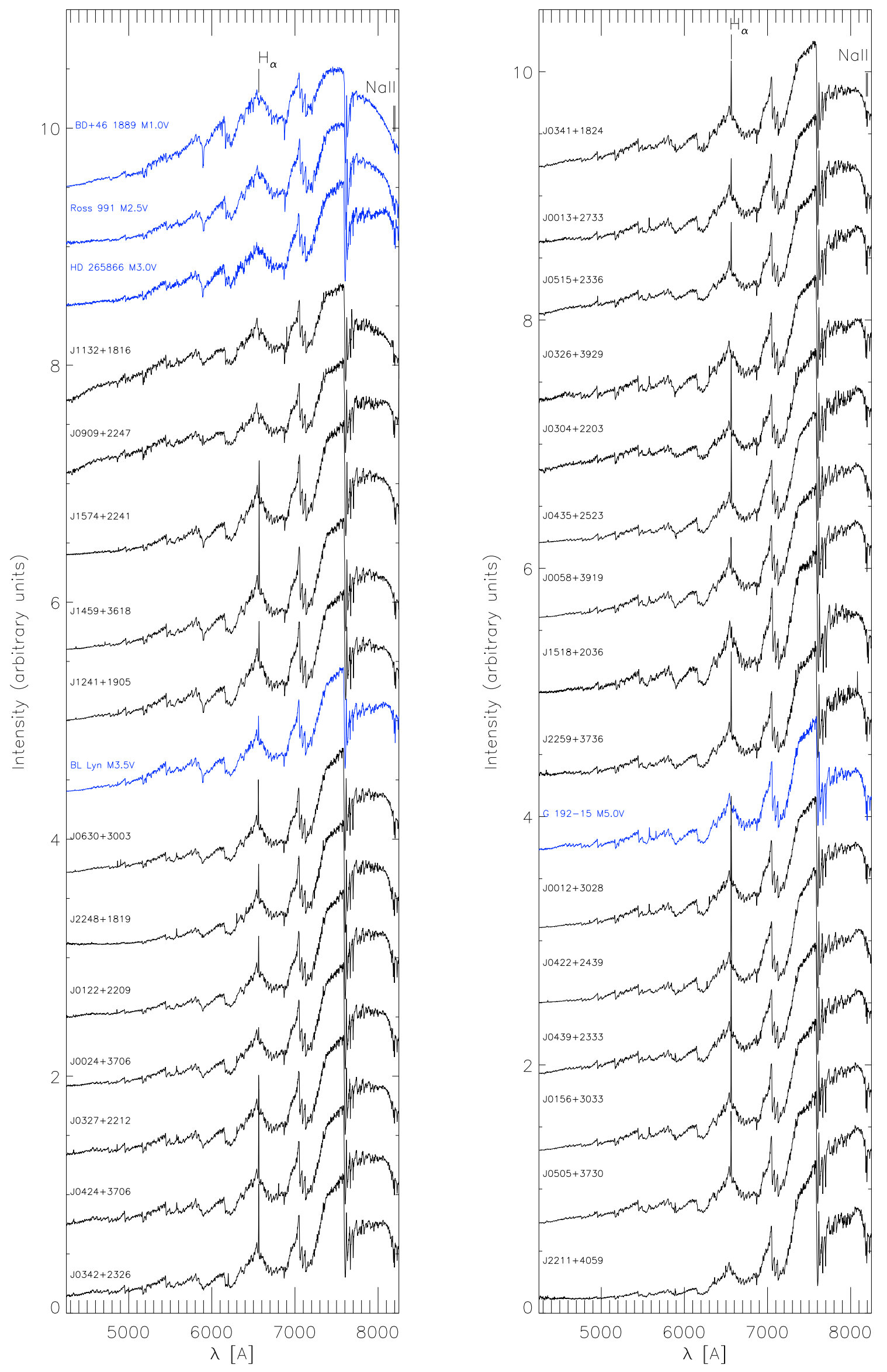

FIG. 2.- IDS/INT spectra of our $27 \mathrm{M}$ dwarf candidates (in black) and five reference stars (in blue), normalized at $7400 \AA$. 
Aberasturi et al.

TABLE 2

Basic Data of the 27 Spectroscopically Analysed M Candidates ${ }^{a}$

\begin{tabular}{|c|c|c|c|c|c|c|c|c|c|}
\hline ID & $\begin{array}{l}\text { Alternative } \\
\text { Name }\end{array}$ & $\alpha(\mathrm{J} 2000)$ & $\delta(\mathrm{J} 2000)$ & $\begin{array}{c}b \\
(\mathrm{deg})\end{array}$ & $\begin{array}{c}\mu_{\alpha} \cos \delta \\
\left(\operatorname{mas~a~}^{-1}\right)\end{array}$ & $\begin{array}{c}\mu_{\delta} \\
\left(\operatorname{mas~a}^{-1}\right)\end{array}$ & $\begin{array}{l}r^{\prime}-J \\
(\mathrm{mag})\end{array}$ & $\begin{array}{c}J-K_{s} \\
(\mathrm{mag})\end{array}$ & $\begin{array}{c}H_{r^{\prime}} \\
(\mathrm{mag})\end{array}$ \\
\hline $\mathrm{J} 0012+3028$ & 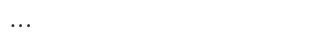 & $00: 12: 13.43$ & $+30: 28: 44.2$ & -31.6 & $+52.7 \pm 5.1$ & $-25.9 \pm 5.1$ & 3.97 & 0.83 & 13.06 \\
\hline $\mathrm{J} 0013+2733$ & $\ldots$ & $00: 13: 19.52$ & $+27: 33: 30.8$ & -34.6 & $+16.3 \pm 4.4$ & $-116.4 \pm 4.4$ & 00 & 85 & 14.79 \\
\hline J0024+2626 & LSPM J0024+2626 & $00: 24: 03.81$ & $+26: 26: 29.7$ & -36.0 & $+7.0 \pm 6.4$ & $-30.1 \pm 6.4$ & 3.90 & 0.92 & 11.58 \\
\hline J0058+3919 & PM I00580+3919 & 00:58:01.13 & $+39: 19: 11.2$ & -23.5 & $-102.6 \pm 9.3$ & $+34.1 \pm 9.3$ & 4 & 38 & 13.77 \\
\hline J0122+2209 & G $34-23$ & $01: 22: 10.32$ & $+22: 09: 03.0$ & -40.2 & $+237.0 \pm 5.3$ & $-152.7 \pm 5.3$ & 3.96 & 87 & 14.63 \\
\hline $\mathrm{J} 0156+3033$ & NLTT 6496 (Kö4A) & $01: 56: 45.76$ & $+30: 33: 28.8$ & -30.2 & $+219.2 \pm 4.6$ & $-12.5 \pm 4.6$ & 4.13 & 37 & 16.16 \\
\hline J0304+2203 & $\ldots$ & 03:04:44.10 & $+22: 03: 21.2$ & -31.2 & $+37.8 \pm 5.1$ & $-49.3 \pm 5.1$ & 4.44 & .83 & 13.90 \\
\hline J0326+3929 & LSPM J0326+3929W & $03: 26: 34.20$ & $+39: 29: 02.5$ & -14.2 & $+61.4 \pm 5.7$ & $-144.1 \pm 5.7$ & 4.00 & 1 & .94 \\
\hline $\mathrm{J} 0327+2212$ & $\ldots$ & $03: 27: 30.87$ & $+22: 12: 38.1$ & -27.9 & $-39.0 \pm 6.3$ & $-53.0 \pm 6.3$ & 4.01 & 0.85 & 13.15 \\
\hline $\mathrm{J} 0341+$ & $\ldots$ & $: 43.87$ & $24: 06.2$ & -28.6 & $+23.9 \pm 4.7$ & $-42.2 \pm 4.7$ & & 34 & 12.84 \\
\hline J0342+2326 & LR Tau & $03: 42: 53.29$ & $+23: 26: 49.5$ & -24.6 & $+176.7 \pm 4.7$ & $-61.2 \pm 4.7$ & 4.06 & 0.89 & 15.63 \\
\hline $\mathrm{J} 0422+2439$ & $\ldots$ & $04: 22: 54.17$ & $+24: 39: 53.6$ & -17.3 & & $-22.3 \pm 4.5$ & 4.88 & 0 & 11.32 \\
\hline J0424+3706 & $\ldots$ & $04: 24: 21.51$ & $+37: 06: 20.8$ & -8.6 & $+47.3 \pm 6.5$ & $-57.6 \pm 6.5$ & 4.01 & 0.85 & 13.56 \\
\hline $5+2523$ & $\ldots$ & 47.79 & $+25: 23: 43.6$ & -14.6 & & $-21.6 \pm 4.5$ & 4.40 & 94 & 11.36 \\
\hline J0439+2333 & $\ldots$ & 04:39:04.54 & $+23: 33: 19.9$ & -15.3 & $-0.9 \pm 4.6$ & $-24.6 \pm 4.6$ & 4.38 & 0.86 & 11.81 \\
\hline $\mathrm{J} 0507+3730$ & $\ldots$ & $05: 07: 14.45$ & $+37: 30: 42.1$ & -1.9 & $-105.5 \pm 5.0$ & $-8.0 \pm 5.0$ & 4.43 & 39 & 14.84 \\
\hline J0515+2336 & $\ldots$ & $05: 15: 17.54$ & $+23: 36: 25.9$ & -8.6 & $+36.7 \pm 4.6$ & $-71.5 \pm 4.6$ & 4.39 & 0.88 & 14.10 \\
\hline $0+3003$ & $\ldots$ & $: 10.18$ & $+30: 03: 39.5$ & +9.0 & $-1.6 \pm 8.2$ & $+28.1 \pm 8.2$ & 3.97 & 0.84 & 11.27 \\
\hline $\mathrm{J} 0909+2247$ & $\ldots$ & 09:09:07.97 & $+22: 47: 41.2$ & +39.8 & $-81.2 \pm 4.2$ & $-71.8 \pm 4.2$ & 4.18 & 0.86 & 14.83 \\
\hline $\mathrm{J} 1132+1816$ & $\ldots$ & $11: 32: 23.00$ & $+18: 16: 22.4$ & +69.8 & $+136.4 \pm 5.5$ & $+58.6 \pm 5.5$ & 3.96 & 0.84 & 14.99 \\
\hline $\mathrm{J} 1241+1905$ & G $59-34$ & $12: 41: 29.00$ & $+19: 05: 00.7$ & +81.6 & $+68.6 \pm 4.9$ & $-305.4 \pm 4.9$ & 3.92 & 0.89 & 16.76 \\
\hline $\mathrm{J} 1459+3618$ & RX J1459.4+3618 & $14: 59: 25.04$ & $+36: 18: 32.3$ & +61.4 & $-123.9 \pm 5.2$ & $+76.1 \pm 5.2$ & 4.03 & 0.88 & 15.10 \\
\hline $\mathrm{J} 1518+2036$ & $\ldots$ & $15: 18: 31.45$ & $+20: 36: 28.3$ & +55.9 & $+10.1 \pm 4.6$ & $+94.8 \pm 4.6$ & 4.67 & 0.85 & 14.68 \\
\hline $\mathrm{J} 1547+2241$ & LSPM J1547+2241 & $15: 47: 40.69$ & $+22: 41: 16.5$ & +50.0 & $-180.9 \pm 5.0$ & $-29.5 \pm 5.0$ & 4.05 & 0.90 & 14.90 \\
\hline $\mathrm{J} 2211+4059$ & 1RXS J221124.3+410000 & $22: 11: 24.14$ & $+40: 59: 58.9$ & -12.4 & $-89.6 \pm 4.9$ & $+68.0 \pm 4.9$ & 4.65 & 0.93 & 14.63 \\
\hline $\mathrm{J} 2248+1819$ & PM I22489+1819 & $22: 48: 54.58$ & $+18: 19: 58.9$ & -35.7 & $-24.7 \pm 5.1$ & $-132.8 \pm 5.1$ & 3.93 & 0.84 & 14.54 \\
\hline $\mathrm{J} 2259+3736$ & $\ldots$ & $22: 59: 14.81$ & $+37: 36: 39.6$ & -20.1 & $+96.2 \pm 4.7$ & $-28.9 \pm 4.7$ & 4.26 & 0.84 & 14.65 \\
\hline
\end{tabular}

${ }^{a}$ Identification, discovery name (blank if new), right ascension and declination from 2MASS, Galactic latitude, proper motions from PPMXL, $r^{\prime}-J$ and $J-K_{s}$ colors, and reduced proper motion $H_{r^{\prime}}=r^{\prime}+5 \log \mu+5$. 
TABLE 3

SPECTRAL-TYPE REFERENCE STARS

\begin{tabular}{|c|c|c|c|c|c|}
\hline Name & GJ & $\alpha(\mathrm{J} 2000)$ & $\delta(\mathrm{J} 2000)$ & $\begin{array}{c}\mathrm{SpT} \\
\mathrm{PMSU}^{a}\end{array}$ & $\begin{array}{c}\text { SpT } \\
\text { Simbad }\end{array}$ \\
\hline G $192-15$ & 3380 & $06: 02: 29.18$ & $+49: 51: 56.2$ & M5.0 V & M $5.0 \mathrm{~V}$ \\
\hline HD 265866 & 251 & $06: 54: 48.96$ & $+33: 16: 05.4$ & M3.0 V & $\mathrm{M} 4.0 \mathrm{~V}$ \\
\hline BL Lyn & $277 \mathrm{~B}$ & $07: 31: 57.33$ & $+36: 13: 47.4$ & M3.5 V & M $4.5 \mathrm{~V}$ \\
\hline Ross 991 & 3748 & $12: 47: 00.99$ & $+46: 37: 33.4$ & $\mathrm{M} 2.5 \mathrm{~V}$ & $\mathrm{M} 2.0 \mathrm{~V}$ \\
\hline $\mathrm{BD}+461889$ & 521 & 13:39:24.10 & $+46: 11: 11.4$ & M1.0 V & $\mathrm{M} 2.0 \mathrm{~V}$ \\
\hline
\end{tabular}
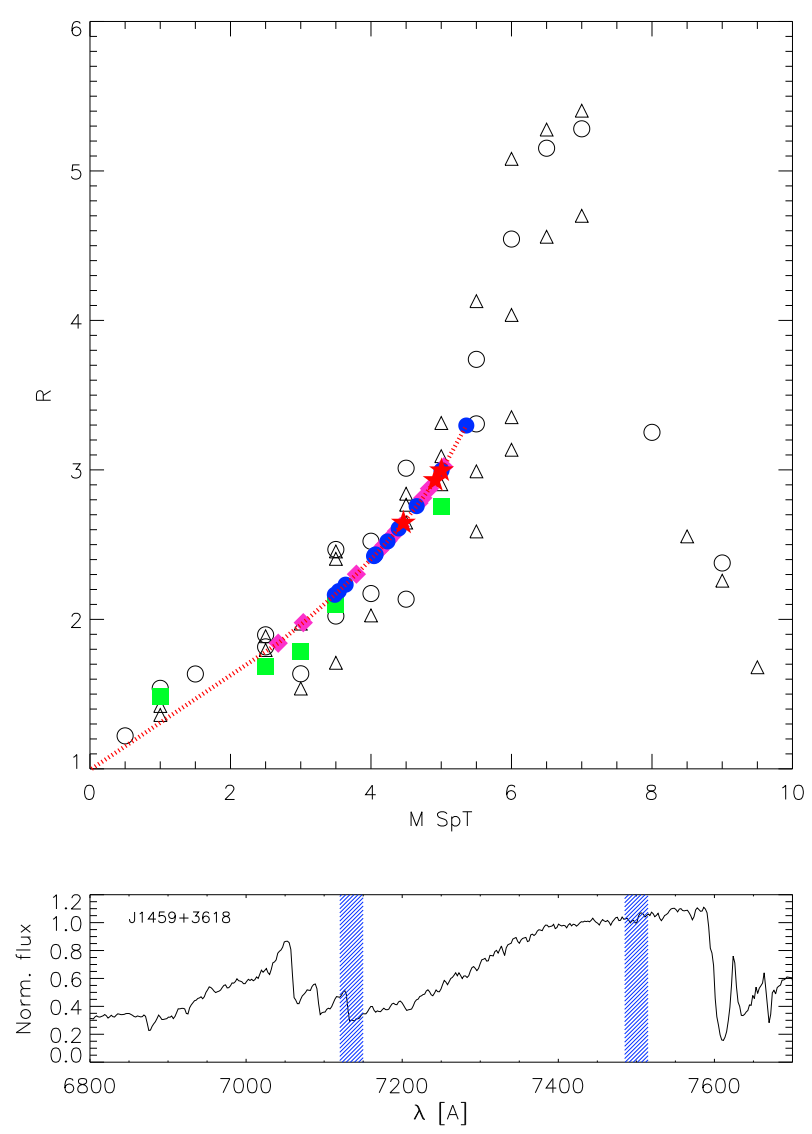

Fig. 3.- Top panel: our spectral index $\Re$ measured for M1$9 \mathrm{~V}$ spectral type templates observed by Leggett et al. (2000) and Cruz \& Reid (2002) (black empty circles and triangles, respectively) compared with our 24 field M dwarfs (blue filled circles) $3 \mathrm{~T}$ Tauri M-type objects (red filled stars), and 5 reference field dwarfs (green filled squares). Bottom panel: wavelength intervals used to define the Re index on an example spectrum.

As explained above, 11 of the 27 high-priority Mdwarf candidates observed spectroscopically were previously known. Of them, seven had spectral-type estimations from colors (from digitization of blue and red photographic plates Giclas et al. 1961; Lépine \& Gaidos 2011- or from optical and near-infrared multi-band photometry Fleming 1998), and two from real spectra (J0122+2209 from Lépine et al. 2013 and J1241+1905 from Reid et al. 2003).

For the 27 stars, we derived our own spectral types with a custom-made spectral index. Spec- tral indices have been extensively used in the classification of M dwarf spectra (Kirkpatrick et al. 1991; Reid et al. 1995; Martín et al. 1996, 1999; Hawlev et al. 2002; Lépine et al. 2003a; $\quad$ Slesnick et al. 2006a, b; Shkolnik etwal. |2011; Seeliger et al. 2011).

We took advantage of this knowledge for defining the $\Re$ index, which better fits the useful wavelength interval, resolution, and maximum efficiency of our IDS/INT spectra. The numerator and denominator of the $\Re$ index are the fluxes contained in the $30 \AA$ bands centered on 7500 and $7135 \AA$, which correspond to the pseudo-continuum at the red side and to the minimum of the strong $~ 7000$ $7350 \AA \mathrm{TiO}$ band, respectively. Basically, $\Re$ is similar to the Martín et al. (1996) PC2 index (7560 $20 \AA$ / $7040 \pm 10 \AA$ ) , which accounts mostly for the TiO and VO contributions, but with the numerator wavelength interval at the bottom of a deep water vapor band head. The $\Re$ index is not sensitive to luminosity, log g or metallicity. To minimize the dependence of our index on flux calibration calibration issues, we normalized our spectra to a pseudo-continuum traced by joining the highest points of the observed spectra (skipping $\mathrm{H} \alpha$ ). The ratios were measured on the "normalized" spectra. As templates for determining the $\Re$-spectral type relation, we used the spectra of 58 M1-9 V stars of Leggett et al. (2000) and Cruz \& Reid (2002), together with our 5 reference stars, normalized in the same way as the target spectra. As illustrated by Figure. 3 , the $\Re$ index has the advantage of having a fairly large dynamic range, covering values from about 1.0 to 5.5 . We fit the $\Re$-SpT pairs to a parabola (i.e., $\operatorname{SpT}(\Re)=a+b \Re+c \Re^{2}$ ), and derived spectral types for our 27 targets with an estimated uncertainty of \pm 0.5 spectral subtypes. The relation to estimate the spectral type, valid between M0.0 V and M5.5 V, was:

$$
S p T=-0.58 \Re^{2}+4.8 \Re-4.2
$$

The results are listed in the third column of Table 4. We calculate 18 new spectral types for the first time and improve previous determinations for other 7 dwarfs. Derived spectral types vary from M2.5 V in the case of J1132+1816 to M5.5 V in the case of J2211+4059 (PM I22489+1819), with the majority of them in the narrow interval from $\mathrm{M} 3.5 \mathrm{~V}$ to $\mathrm{M} 4.5 \mathrm{~V}$, which fully backs our initial color criteria for selecting intermediate $\mathrm{M}$ dwarfs (the original cut in $r^{\prime}-J$ was for selecting $>\mathrm{M} 4 \mathrm{~V}$ stars). Some of the stars M4 V and later are bright enough to be potential targets for exoplanet surveys, such as CARMENES Quirrenbach et al. 2012; Caballero et al. 2013). Interestingly, the latest $\mathrm{M}$ dwarf in our sample had a spectral-type estimation from $V_{\text {phot }}-J$ photometry at $\mathrm{M} 7: \mathrm{V}$. We agree with Lépine et al. (2013) and Mundt et al. (2013) that spectral types from this color are systematically later than those actually measured on real spectra (at least for spectral types later than M2-3 V).

\subsection{Effective Temperatures and Surface Gravities}

We used another VO tool, the VO Spectral energy distribution Analyzer (VOSA6; Bayo et al. 2008), to derive effective temperatures $\left(T_{\text {eff }}\right)$ and surface gravities $(\log g)$

\footnotetext{
${ }^{6}$ http://svo2.cab.inta-csic.es/theory/vosa/
} 
TABLE 4

Miscellaneous Data of the 27 Investigated Stars ${ }^{a}$

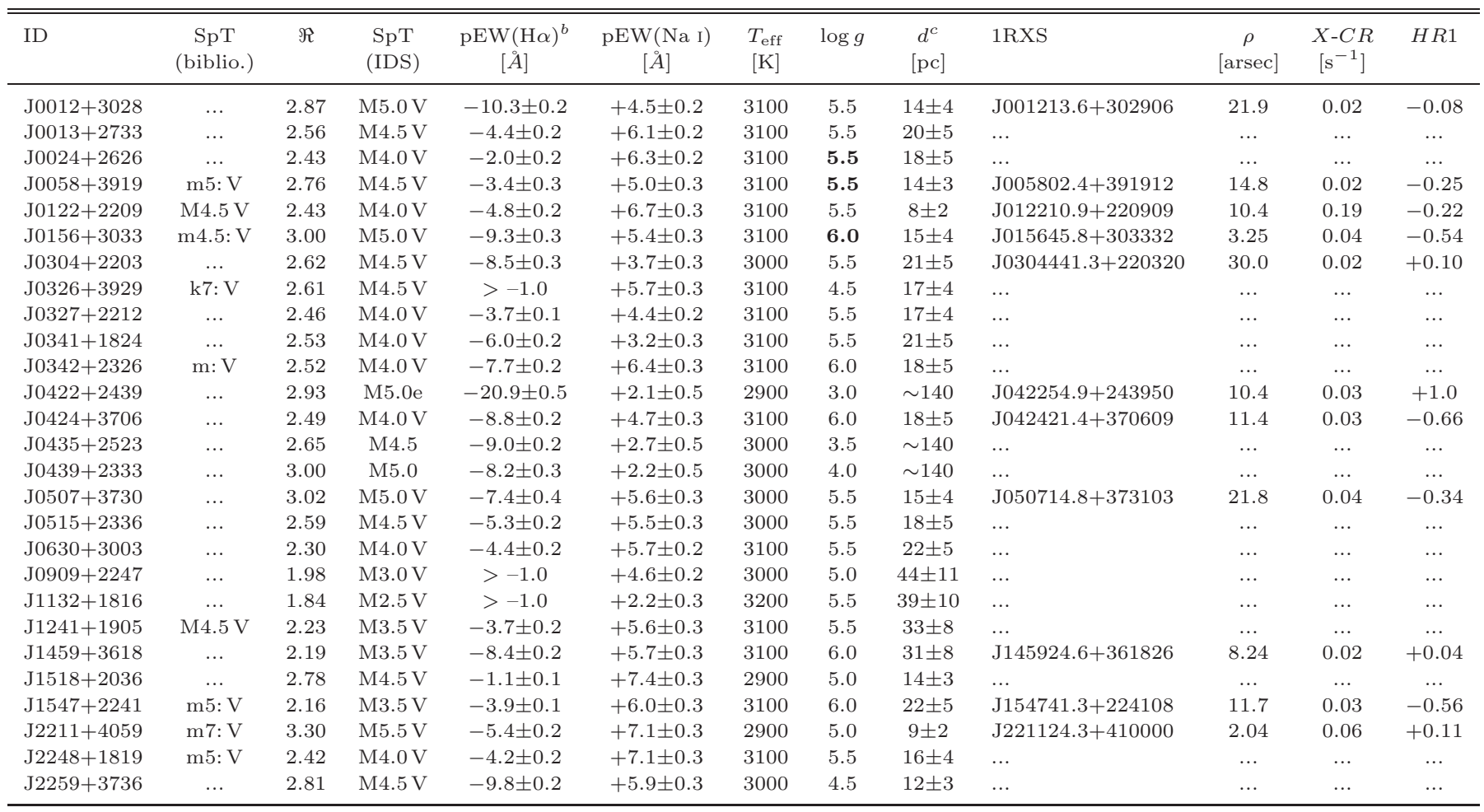

${ }^{a}$ Spectral types from the bibliography and our IDS/INT spectra (photometric spectral types are listed with ' $\mathrm{m}$ ' and ' $\mathrm{k}$ '), $\Re$ index, pseudo-equivalent widths of $\mathrm{H} \alpha \lambda 656.3 \mathrm{~nm}$ and $\mathrm{Na}$ I $\lambda \lambda 818.3,819.5 \mathrm{~nm}$ from our spectra, $T_{\text {eff }}( \pm 100 \mathrm{~K})$ and $\log g( \pm 0.5)$ from our VOSA fits, derived heliocentric distance, and key data from the ROSAT All-Sky Bright and Faint Survey Catalogues (1RXS name, angular separation between the X-ray and 2MASS coordinates, count rate, and hardness ratio).

${ }^{b}$ Two stars had previous pEW(H $\alpha$ ) determinations: J0122+2209 of $-4.1 \pm 0.7 \AA$ and J1459+3618 of $-6.9 \pm 0.6 \AA$ (Mochnacki et al. 2002$)$.

${ }^{c}$ Three stars had previous distance determinations: J0122+2209 at $10.5 \mathrm{pc}$ and J1459+3618 at $22.0 \mathrm{pc}$ (Fleming 1998), J0156+3033 at $19_{-4}^{+6} \mathrm{pc}$ Caballero 2012).

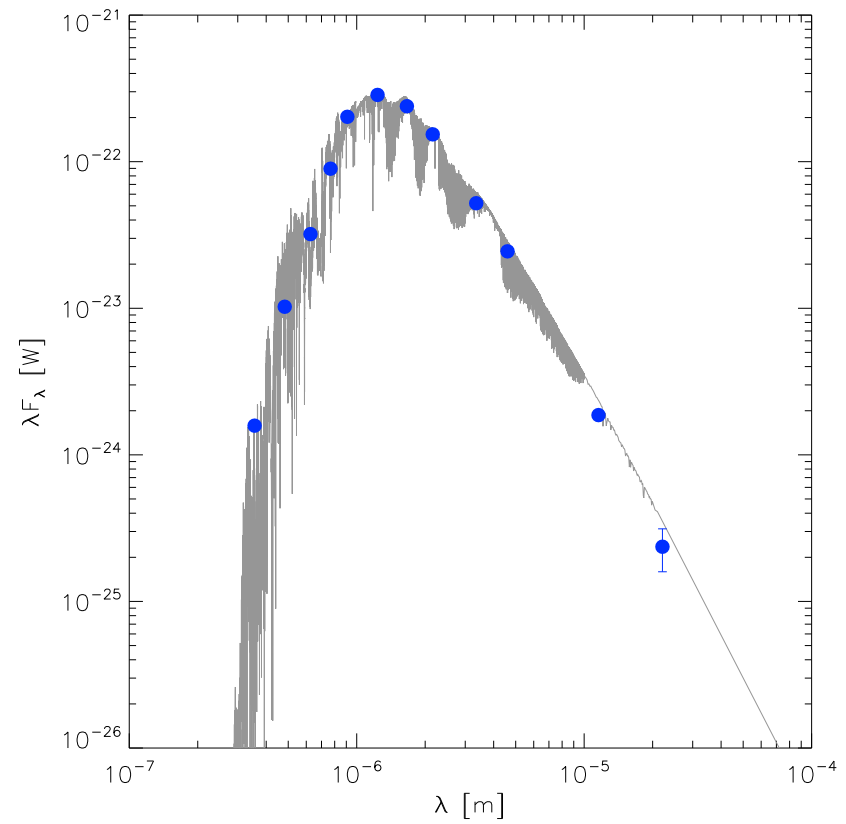

FIG. 4.- Spectral energy distribution of J1459+3618 from $350 \mathrm{~nm}\left(u^{\prime}\right)$ to $22,100 \mathrm{~nm}(W 4)$, in blue filled circles, and the corresponding best-fit NextGen model provided by VOSA. of our 27 targets from fits of observed spectral energy distributions to theoretical models. Apart from the CMC14 $\left(r^{\prime}\right)$ and 2MASS $\left(J H K_{s}\right)$ photometric data, we also used those of the Wide-field Infrared Survey Explorer (W14; WISE, Cutri \& et al. 2012) and, when available, the Fourth U. S. Naval Observatory CCD Astrograph Catalogue ( $B g^{\prime} V i^{\prime}$; UCAC4, Zacharias et al. 2012), and Sloan Digital Sky Survey ( $u^{\prime} g^{\prime}$; SDSS DR9, Aihara et al. 2011). We did not use the Hartman et al. (2011) $V$-band photometry of J1459+3618/RX J1459.4+3618 because of an incorrect absolute calibration (besides, they found a period of photometric variability of $4.17 \mathrm{~d}$, but with a $V$ band amplitude of only $49 \mathrm{mmag})$. Key photometry of the 27 targets is provided in Table 5 .

In VOSA, we used the BT-Settl theoretical models Allard 2014 between 1600 and $4000 \mathrm{~K}$ in $T_{\text {eff }}$ and between 3.5 and 6.0 in $\log g$ for solar metallicity. The uncertainty in the best fit was the size of the grid, which was of $100 \mathrm{~K}$ in $T_{\text {eff }}$ and 0.5 in $\log g$. Anayway, the VOSA $\log g$ values have to be taken with caution and refined using other indicators. Figure. 4 illustrates one of our VOSA fits as an example.

Derived values ranged between 2800 and $3400 \mathrm{~K}$ in $T_{\text {eff }}$, and 3.5 and 6.0 in $\log g$, which roughly match our spectral types and the surface gravity expected values for normal mid-M dwarfs in the field (e.g., Rajpurohit et al. 2013). 
As a matter of fact, the star with the latest spectral type in our sample (J2211+4059, M5.5 V) also had the lowest effective temperature $\left(T_{\text {eff }}=2900 \pm 100 \mathrm{~K}\right)$.

We double-checked the VOSA values of $\log g$ with an atomic gravity-sensitive feature present in our spectra, the Na I doublet at $818.3-819.5 \mathrm{~nm}$ (Steele \& Jameson 1995; Guieu et al. 2006; Slesnick et al. 2006a; Martín et al. 2010; Schlieder et al. 2012). At a given spectral type in low-mass stars (and brown dwarfs), the weaker the alkali doublet, the lower the gravity. In its turn, low gravity is an indicator of youth (Béiar et al. 1999; McGovern et al. 2004; Burningham et al. 2005; Soderblom et al. 2013 and references therein). We measured the pseudo-equivalent widths of the alkali doublet in our IDS/INT spectra with the IRAF task splot. The results, given in Table 4 showed that there are three M4.0-5.0 stars with significantly weak sodium absorption, of $\mathrm{pEW}(\mathrm{Na} \mathrm{I})<3 \AA$; two of them also had the lowest surface gravities $(\log g=3.5 \pm 0.5)$ in the VOSA fits. The other 24 stars had sodium absorptions typical of field dwarfs of the same spectral types (see Table 4 in Schlieder et al. 2012).

The compiled photometry also allowed us to search for infrared excesses, which may be ascribed to circumstellar disks. In particular, two stars, J0515+2336 and $\mathrm{J} 0507+3730$, had a significantly bright $W 4$ magnitude (at $22.1 \mu \mathrm{m}$ ) with respect to the other WISE and 2MASS magnitudes (Table 51). However, their apparent W4-band excess came instead from an incorrect background subtraction at very low Galactic latitudes (column $b$ in Table 2). All in all, no star in our sample displayed a clear mid-infrared flux excess attributable to a disks.

\subsection{Activity}

We tried to quantify the magnetic activity of the stars in our sample. First, we measured pseudo-equivalent widths of the $\mathrm{H} \alpha \lambda 656.3 \mathrm{~nm}$ line, $\mathrm{pEW}(\mathrm{H} \alpha) \mathrm{s}$, in our IDS/INT spectra. Error bars for each target were assigned by manual repetition of measurements making educated visual inspections of the continuum levels and the line limits. As expected for intermediateand late-type M dwarfs (Hawlev et al. 1996; Gizis et al. 2000; West et al. 2004), most of our stars showed $\mathrm{H} \alpha$ in emission. Indeed, two of the three non- $\mathrm{H} \alpha$ emitters are the earliest stars in our sample (M2.5-3.0 V). However, one of the stars displayed an $\mathrm{H} \alpha$ pseudo-equivalent width that stood out among the other measurements: J0422+2439, with pEW $(\mathrm{H} \alpha)=-20.9 \pm 0.5 \AA$. We used the Barrado y Navascués \& Martín (2003) empirical criterion for ascertaining the origin of the $\mathrm{H} \alpha$ emission. As illustrated by Figure. 5 the emission of all stars in our sample except J0422+2439 is consistent with chromospheric activity. J0422+2439, one of the three lowgravity stars described in Section 3.2. showed $\mathrm{H} \alpha$ emission very close to the criterion boundary separating accretion and chromospheric emission.

There were two stars, J0122+2209/G 34-53 and $\mathrm{J} 1459+3618 / \mathrm{RX} \mathrm{J} 1459.4+3618$, for which $\mathrm{H} \alpha$ emission had been investigated previously by Mochnacki et al. (2002) (Table 44). Their and our measurements of $\mathrm{pEW}(\mathrm{H} \alpha)$ match each other within the uncertainties.

Second, we searched for counterparts in the ROSAT All-Sky Bright and Faint Survey Catalogues (Voges et al.

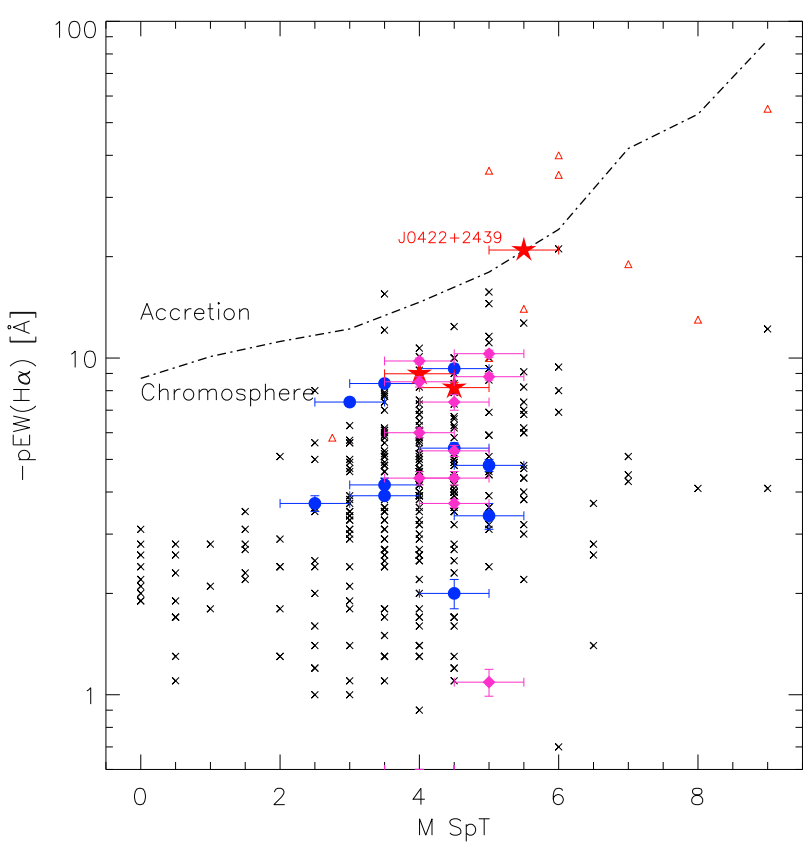

FIG. 5.- Pseudo-equivalent widths of $\mathrm{H} \alpha$ as a function of spectral type with the Barrado y Navascués \& Martín (2003) accretionchromosphere boundary. Small (black) crosses are field M dwarfs from Gershberg et al. (1999), (red) triangles are confirmed TaurusAuriga members from Martín et al. (2001), Luhman et al. (2003), and Muzerolle et al. (2003), (magenta and blue) filled circles are the 24 (new and known) field stars investigated here, and (red) filled stars are our three new Taurus-Auriga member candidates, including J0422+2439.

1999). We applied a search radius of 30 arcsec due to the low ROSAT astrometric precision. Of the 27 stars in our sample, 11 stars $(\sim 40 \%)$ had appreciable emission in the $0.2-2.0 \mathrm{keV}$ energy band at the time of the ROSAT observations.

The brightest star in our sample in the visible and near-infrared, J0122+2209/G 34-53, also has the highest X-ray count rate by far. Perhaps because of that reason, it has been the subject of a few all-sky X-ray surveys for low-mass stars (Fleming 1998; Zickgraf et al. 2003; Fuhrmeister \& Schmitt 2003; Haakonsen \& Rutledge 2009). Another three known dwarfs had also been cataloged as Xray emitters: J0156+3033/NLTT 6896 (Caballero 2012), J1459+3618/RX J1459.4+3618 (Fleming 1998), and J2211+4059/1RXS J221124.3+410000 (Haakonsen \& Rutledge 2009). In this work, we report for the first time the X-ray emission of one known M dwarf, J0058+3919/PM I00580+3919, and six new M dwarfs.

For the $11 \mathrm{X}$-ray stars, we calculated the distanceindependent parameter $F_{X} / F_{J}\left(\equiv L_{X} / L_{J}\right)$, which is a proxy of $L_{X} / L_{\text {bol }}$ (Caballero et al. 2010). We computed $F_{X}$ from the X-ray count rates and hardness ratios as in Schmitt et al. (1995)). Six stars had $F_{X} / F_{J}$ ratios above $0.7 \times 10^{-3}$, with only one of them having a ratio of $\sim 1.1 \times 10^{-3}$. This relatively strong X-ray emitter is just J2211+4059/1RXS J221124.3+410000, the primary of an ultra fragile binary system. The other five intense emitters are J0122+2209/G 34-53 (the brightest star of our list), J0156+3033/NLTT 6896 (the primary of the 
TABLE 5

Photometry of the 27 investigated stars

\begin{tabular}{|c|c|c|c|c|c|c|}
\hline ID & $\begin{array}{c}u^{\prime} \\
{[\mathrm{mag}]}\end{array}$ & $\begin{array}{c}B \\
{[\mathrm{mag}]}\end{array}$ & $\begin{array}{c}g^{\prime} \\
{[\mathrm{mag}]}\end{array}$ & $\begin{array}{c}V \\
{[\mathrm{mag}]}\end{array}$ & $\begin{array}{c}r^{\prime} \\
{[\mathrm{mag}]}\end{array}$ & $\begin{array}{c}J \\
{[\mathrm{mag}]}\end{array}$ \\
\hline $\mathrm{J} 0012+3028$ & $17.884 \pm 0.013$ & $16.361 \pm 0.180$ & $15.801 \pm 0.004$ & $14.864 \pm 0.060$ & 14.216 & $10.242 \pm 0.023$ \\
\hline $\mathrm{J} 0013+2733$ & $19.224 \pm 0.039$ & $16.753 \pm 0.080$ & $16.012 \pm 0.003$ & $15.115 \pm 0.060$ & 14.437 & $10.431 \pm 0.020$ \\
\hline J0024+2626 & $18.122 \pm 0.018$ & $16.343 \pm 0.130$ & $15.550 \pm 0.003$ & $14.735 \pm 0.040$ & 14.131 & $10.222 \pm 0.020$ \\
\hline J0058+3919 & $17.552 \pm 0.01$ & $15.837 \pm 0.050$ & $15.078 \pm 0.003$ & $14.168 \pm 0.030$ & 13.598 & $9.561 \pm 0.026$ \\
\hline J0122+2209 & $\ldots$ & $14.722 \pm 0.030$ & $13.763 \pm 0.04$ & $12.996 \pm 0.030$ & 12.375 & $8.412 \pm 0.021$ \\
\hline J0156+3033 & $\ldots$ & $16.532 \pm 0.050$ & $15.786 \pm 0.08$ & $15.105 \pm 0.050$ & 14.449 & $10.323 \pm 0.023$ \\
\hline $\mathrm{J} 0304+2203$ & & $17.164 \pm 0.030$ & $16.199 \pm 0.05$ & $15.564 \pm 0.030$ & 14.931 & $10.486 \pm 0.022$ \\
\hline $\mathrm{J} 0326+3929^{b}$ & $17.991 \pm 0.013$ & & $15.546 \pm 0.003$ & & 13.968 & $9.998 \pm 0.025$ \\
\hline $\mathrm{J} 0327+2212$ & $\ldots$ & $16.252 \pm 0.120$ & $15.314 \pm 0.04$ & $14.654 \pm 0.090$ & 14.055 & $10.044 \pm 0.022$ \\
\hline J0341+1824 & $\ldots$ & $16.577 \pm 0.030$ & $15.669 \pm 0.03$ & $14.958 \pm 0.100$ & 14.412 & $10.484 \pm 0.021$ \\
\hline $\mathrm{J} 0342+2326$ & & $16.609 \pm 0.060$ & $15.667 \pm 0.05$ & $14.984 \pm 0.060$ & 14.267 & $10.202 \pm 0.022$ \\
\hline $\mathrm{J} 0422+2439^{b}$ & $18.426 \pm 0.014$ & & $16.092 \pm 0.003$ & & 14.529 & $9.648 \pm 0.021$ \\
\hline J0424+3706 & $17.711 \pm 0.012$ & $16.337 \pm 0.020$ & $15.718 \pm 0.005$ & $14.668 \pm 0$ & 14.203 & $10.191 \pm 0.026$ \\
\hline $\mathrm{J} 0435+2523^{b}$ & $18.303 \pm 0.014$ & & $16.189 \pm 0.004$ & & 14.669 & $10.272 \pm 0.022$ \\
\hline J0439+2333 & $18.433 \pm 0.015$ & $17.028 \pm 0.120$ & $16.240 \pm 0.004$ & $15.392 \pm 0.010$ & 14.856 & $10.479 \pm 0.023$ \\
\hline $\mathrm{J} 0507+3730$ & $\ldots$ & $16.986 \pm 0.230$ & $16.018 \pm 0.03$ & $15.349 \pm 0.060$ & 14.713 & $10.284 \pm 0.020$ \\
\hline $\mathrm{J} 0515+2336$ & $\ldots$ & $16.977 \pm 0.010$ & $16.031 \pm 0.05$ & $15.245 \pm 0.040$ & 14.574 & $10.186 \pm 0.030$ \\
\hline $\mathrm{J} 0630+3003$ & & & & & 14.020 & $10.045 \pm 0.018$ \\
\hline $\mathrm{J} 0909+2247$ & $18.856 \pm 0.018$ & $17.172 \pm 0.010$ & $16.230 \pm 0.004$ & $15.266 \pm 0.010$ & 14.656 & $10.474 \pm 0.023$ \\
\hline $\mathrm{J} 1132+1816$ & $18.223 \pm 0.016$ & $16.354 \pm 0.070$ & $15.625 \pm 0.004$ & $14.751 \pm 0.050$ & 14.137 & $10.175 \pm 0.023$ \\
\hline $\mathrm{J} 1241+1905^{b}$ & $18.369 \pm 0.017$ & $16.750 \pm 0.080$ & $15.882 \pm 0.004$ & $15.048 \pm 0.030$ & 14.284 & $10.368 \pm 0.022$ \\
\hline $\mathrm{J} 1459+3618$ & $18.183 \pm 0.012$ & $16.566 \pm 0.040$ & $15.784 \pm 0.004$ & $14.845 \pm 0.080$ & 14.292 & $10.257 \pm 0.018$ \\
\hline $\mathrm{J} 1518+2036$ & & & $\ldots$ & $15.455 \pm 0.010$ & 14.786 & $10.119 \pm 0.021$ \\
\hline $\mathrm{J} 1574+2241$ & $17.438 \pm 0.013$ & $15.955 \pm 0.040$ & $15.139 \pm 0.004$ & $14.197 \pm 0.010$ & 13.589 & $9.543 \pm 0.022$ \\
\hline $\mathrm{J} 2211+4059$ & $18.465 \pm 0.015$ & $16.963 \pm 0.090$ & $16.081 \pm 0.003$ & $15.139 \pm 0.010$ & 14.371 & $9.725 \pm 0.020$ \\
\hline $\mathrm{J} 2248+1819$ & $17.838 \pm 0.011$ & $16.115 \pm 0.080$ & $15.461 \pm 0.005$ & $14.535 \pm 0.040$ & 13.886 & $9.957 \pm 0.021$ \\
\hline $\mathrm{J} 2259+3736$ & & $16.942 \pm 0.020$ & $16.043 \pm 0.05$ & $15.357 \pm 0.120$ & 14.638 & $10.378 \pm 0.029$ \\
\hline ID & $\left.\begin{array}{c}H \\
{[\mathrm{mag}]}\end{array}\right]$ & $\begin{array}{c}K_{\mathrm{s}} \\
{[\mathrm{mag}]}\end{array}$ & $\begin{array}{c}W 1 \\
{[\mathrm{mag}]}\end{array}$ & $\begin{array}{c}W 2 \\
{[\mathrm{mag}]}\end{array}$ & $\begin{array}{c}W 3 \\
{[\mathrm{mag}]}\end{array}$ & $\begin{array}{c}W 4 \\
{[\mathrm{mag}]}\end{array}$ \\
\hline $\mathrm{J} 0012+3028$ & $9.683 \pm 0.022$ & $9.410 \pm 0.021$ & $9.233 \pm 0.022$ & $9.039 \pm 0.020$ & $8.929 \pm 0.026$ & $8.784 \pm 0.386$ \\
\hline+2733 & $9.837 \pm 0.020$ & $9.581 \pm 0.018$ & $9.376 \pm 0.024$ & $9.210 \pm 0.022$ & $9.077 \pm 0.027$ & $8.592 \pm 0.317$ \\
\hline J0024+2626 & $9.592 \pm 0.019$ & $9.299 \pm 0.017$ & $9.188 \pm 0.023$ & $8.959 \pm 0.020$ & $8.819 \pm 0.025$ & $8.543 \pm 0.298$ \\
\hline J0058+3919 & $8.947 \pm 0.029$ & $8.680 \pm 0.018$ & $8.489 \pm 0.023$ & $8.303 \pm 0.020$ & $8.150 \pm 0.017$ & $8.009 \pm 0.133$ \\
\hline J0122+2209 & $7.820 \pm 0.016$ & $7.537 \pm 0.017$ & $7.341 \pm 0.027$ & $7.167 \pm 0.020$ & $7.055 \pm 0.016$ & $6.856 \pm 0.060$ \\
\hline J0156+3033 & $9.718 \pm 0.031$ & $9.449 \pm 0.021$ & $9.267 \pm 0.023$ & $9.074 \pm 0.019$ & $8.925 \pm 0.028$ & $9.075 \pm 0.540$ \\
\hline $\mathrm{J} 0304+2203$ & $9.933 \pm 0.021$ & $9.655 \pm 0.018$ & $9.427 \pm 0.023$ & $9.226 \pm 0.022$ & $9.098 \pm 0.032$ & 8.476: ${ }^{a}$ \\
\hline $\mathrm{J} 0326+3929^{b}$ & $9.412 \pm 0.026$ & $9.084 \pm 0.017$ & $8.884 \pm 0.028$ & $8.701 \pm 0.026$ & $8.616 \pm 0.030$ & $8.017 \pm 0.274$ \\
\hline $\mathrm{J} 0327+2212$ & $9.477 \pm 0.020$ & $9.194 \pm 0.017$ & $9.077 \pm 0.022$ & $8.872 \pm 0.020$ & $8.730 \pm 0.029$ & $8.812 \pm 0.434$ \\
\hline J0341+1824 & $9.858 \pm 0.023$ & $9.643 \pm 0.023$ & $9.435 \pm 0.023$ & $9.245 \pm 0.021$ & $9.175 \pm 0.036$ & $8.866:^{a}$ \\
\hline J0342+2326 & $9.545 \pm 0.023$ & $9.316 \pm 0.023$ & $9.158 \pm 0.025$ & $8.979 \pm 0.022$ & $8.817 \pm 0.028$ & $8.076 \pm 0.354$ \\
\hline $\mathrm{J} 0422+2439^{b}$ & $8.947 \pm 0.022$ & $8.651 \pm 0.020$ & $8.467 \pm 0.032$ & $8.185 \pm 0.030$ & $8.162 \pm 0.028$ & $8.014 \pm 0.269$ \\
\hline J0424+3706 & $9.553 \pm 0.030$ & $9.340 \pm 0.020$ & $9.184 \pm 0.023$ & $9.009 \pm 0.019$ & $8.830 \pm 0.030$ & $8.193:^{a}$ \\
\hline $\mathrm{J} 0435+2523^{b}$ & $9.618 \pm 0.030$ & $9.331 \pm 0.021$ & $9.216 \pm 0.023$ & $8.997 \pm 0.020$ & $8.821 \pm 0.027$ & $8.249 \pm 0.262$ \\
\hline J0439+2333 & $9.893 \pm 0.021$ & $9.617 \pm 0.017$ & $9.471 \pm 0.024$ & $9.258 \pm 0.020$ & $9.131 \pm 0.033$ & $8.530 \pm 0.381$ \\
\hline $\mathrm{J} 0507+3730$ & $9.703 \pm 0.021$ & $9.397 \pm 0.018$ & $9.223 \pm 0.022$ & $9.008 \pm 0.019$ & $8.686 \pm 0.024$ & $7.656 \pm 0.133$ \\
\hline $\mathrm{J} 0515+2336$ & $9.602 \pm 0.035$ & $9.306 \pm 0.024$ & $9.067 \pm 0.024$ & $8.897 \pm 0.020$ & $8.672 \pm 0.026$ & $7.397 \pm 0.175$ \\
\hline $\mathrm{J} 0630+3003$ & $9.484 \pm 0.018$ & $9.208 \pm 0.018$ & $9.025 \pm 0.023$ & $8.847 \pm 0.018$ & $8.700 \pm 0.029$ & $8.090 \pm 0.256$ \\
\hline J0909+2247 & $9.915 \pm 0.032$ & $9.616 \pm 0.018$ & $9.367 \pm 0.021$ & $9.206 \pm 0.020$ & $8.996 \pm 0.030$ & $8.470:^{a}$ \\
\hline J1132+1816 & $9.599 \pm 0.030$ & $9.338 \pm 0.022$ & $9.138 \pm 0.024$ & $8.961 \pm 0.021$ & $8.821 \pm 0.024$ & $9.182 \pm 0.534$ \\
\hline $\mathrm{J} 1241+1905^{b}$ & $9.792 \pm 0.026$ & $9.477 \pm 0.018$ & $9.314 \pm 0.023$ & $9.136 \pm 0.019$ & $8.978 \pm 0.025$ & $8.336 \pm 0.225$ \\
\hline $\mathrm{J} 1459+3618$ & $9.647 \pm 0.016$ & $9.377 \pm 0.016$ & $9.230 \pm 0.022$ & $9.070 \pm 0.020$ & $8.939 \pm 0.021$ & $9.123 \pm 0.353$ \\
\hline $\mathrm{J} 1518+2036$ & $9.606 \pm 0.022$ & $9.268 \pm 0.019$ & $9.039 \pm 0.022$ & $8.839 \pm 0.020$ & $8.641 \pm 0.019$ & $8.515 \pm 0.194$ \\
\hline $\mathrm{J} 1574+2241$ & $8.932 \pm 0.030$ & $8.647 \pm 0.022$ & $8.475 \pm 0.023$ & $8.305 \pm 0.020$ & $8.145 \pm 0.017$ & $8.007 \pm 0.157$ \\
\hline $\mathrm{J} 2211+4059$ & $9.097 \pm 0.017$ & $8.790 \pm 0.016$ & $8.565 \pm 0.021$ & $8.404 \pm 0.020$ & $8.213 \pm 0.019$ & $8.661 \pm 0.304$ \\
\hline $\mathrm{J} 2248+1819$ & $9.388 \pm 0.020$ & $9.119 \pm 0.017$ & $8.945 \pm 0.022$ & $8.760 \pm 0.020$ & $8.604 \pm 0.023$ & $8.523:^{a}$ \\
\hline $\mathrm{J} 2259+3736$ & $9.890 \pm 0.037$ & $9.535 \pm 0.024$ & $0.257 \pm 0.023$ & $9.062 \pm 0.020$ & $8.925 \pm 0.025$ & $8.509 \pm 0.264$ \\
\hline
\end{tabular}

${ }^{a}$ Poor quality flags.

${ }^{b}$ The SDSS $i^{\prime}$ band was not use to estimated the $T_{\text {eff }}$ 
Koenigstuhl 4 wide binary system), J0422+2439 (the accreting star with strong $\mathrm{H} \alpha$ emission and low surface gravity), and J0304+2203 and J0507+3730 (two new, M4.5-5.0 V stars identified in this work). The latter three intense X-ray emitters are shown here for the first time. The other six stars with ROSAT data are relatively faint X-ray emitters.

\subsection{Three New Member Candidates in Taurus}

The strong $\mathrm{H} \alpha$ emission, low surface gravity, and relatively intense X-ray emission of the possibly accreting star J0422+2439 led us to investigate it in detail. Its coordinates, as well as those of the other two stars with weak $\mathrm{Na}$ I absorption, resembled those of the four known young $\mathrm{T}$ Tauri stars in Table 1. so we considered their membership in the young star-forming region of TaurusAuriga $(\tau \sim 1-2 \mathrm{Ma}, d \sim 140 \mathrm{pc}$; Kenvon et al. 2008).

First of all, we compiled an exhaustive list of TaurusAuriga members and member candidates from Luhman (2004), Luhman et al. (2006, 2009), and Guieu et al. (2006) and cross-matched that list with 2MASS, CMC14, and PPMXL. Figure. 6 illustrates our analyses. From left to right, our three new Taurus-Auriga member candidates with spectroscopy (and the four $\mathrm{T}$ Tauri stars in Table 10 (1) are spatially located towards the densest filaments of Taurus-Auriga (see also Figure 1 in Luhman et al. 2009), (2) have proper motions consistent with membership in Taurus-Auriga (Bertout \& Genova 2006; Mooley et al. 2013), and (3) follow the TaurusAuriga sequence in optical-near-infrared color-magnitude diagrams7. Taking into account these facts, the low surface gravity of the three new member candidates, and the intense $\mathrm{H} \alpha$ and X-ray emission of the accreting star, we concluded that J0422+2439, J0435+2523, and J0439+2333 do belong to Taurus-Auriga.

We further characterized the three identified $\mathrm{T}$ Tauri stars. At $d \sim 140 \mathrm{pc}$, the Taurus-Auriga distance modulus is $m-M \sim 5.7$ mag. With the $J$-band apparent magnitudes, the maximum absolute magnitudes range between 3.9 and $4.7 \mathrm{mag}$. Actual absolute magnitudes must be brighter than that interval because of variable extinction toward Taurus-Auriga. The detection of J0422+2439 by ROSAT implies that it is located in the closer side of the cloud, with subsequent low extinction (X-rays are absorbed by interstellar dust and gas). Something similar happens to J0435+2523 and J0439+2333, whose SEDs and colors do not deviate significantly from the other $\mathrm{M}$ dwarfs in the field. By assuming conservatively that the $J$-band extinction is lower than $0.5 \mathrm{mag}$ and using the NextGen models (Baraffe et al. 1998) at 1$2 \mathrm{Ma}$, we derived theoretical masses in the interval 0.17 $0.57 M_{\odot}$ for the three new $\mathrm{T}$ Tauri low-mass stars, well above the hydrogen burning mass limit. The three young stars could be at distances slightly closer than $140 \mathrm{pc}$ because they are on the near sides of the clouds, which are rather large. We will know their distances with the advent of the ESA/Gaia/ space mission.

Some authors have argued against the universality of the initial mass function based on the hypothetical deficit

\footnotetext{
${ }^{7}$ Note the previously known Taurus-Auriga candidates with discordant proper motions or blue $r^{\prime}-J$ colors for their $r^{\prime}$ magnitudes (CFHT-BD-Tau 19, KPNO-Tau 6, 2MASS J04201611+2821325, 2MASS J04202144+2813491, ITG 34 and FS 115.
}

TABLE 6

Relative Astrometry of the LSPM J0326+3929EW (Koenigstuhl 7 AB) Common Proper Motion Pair

\begin{tabular}{cccl}
\hline \hline $\begin{array}{c}\rho \\
\text { arcsec }]\end{array}$ & $\begin{array}{c}\theta \\
{[\mathrm{deg}]}\end{array}$ & Date & Origin \\
\hline $6.5 \pm 0.5$ & $236 \pm 6$ & 1955 Feb 13 & POSS-I Red \\
$5.7 \pm 0.5$ & $229 \pm 6$ & 1989 Sep 29 & POSS-II Red \\
$6.2 \pm 0.5$ & $229 \pm 6$ & 1994 Nov 28 & POSS-II Blue \\
$5.9 \pm 0.5$ & $228 \pm 6$ & 1995 Nov 14 & POSS-II IR \\
$6.37 \pm 0.06$ & $227.7 \pm 1.0$ & 1998 Nov 1 & 2MASS \\
$6.38 \pm 0.10$ & $227.3 \pm 1.0$ & 2001 Dec 26 & CMC14 \\
$6.45 \pm 0.09$ & $227.4 \pm 1.0$ & 2003 Jan 7 & SDSS-DR9 \\
$6.70 \pm 0.11$ & $229.4 \pm 1.0$ & 2010 Jul 1 & WISE \\
\hline
\end{tabular}

of low-mass stellar objects $\left(<0.5 M_{\odot}, \mathrm{M} 2-4\right)$ in TaurusAuriga (Briceño et al. 1998, 2002; Luhman et al. 2003, 2009; Guieu et al. 2006; Güdel et al. 2007; Scelsi et al. 2007; (Perger et al. 2013). The detection of three stars just in the deficit range may point out that the initial mass function in Taurus-Auriga is actually standard, and that previous surveys had not been successful enough for detecting intermediate M dwarfs. Simple VO surveys such as the one presented here may cover that gap.

\subsection{Distances}

The three young stars in Taurus-Auriga are consequently located at $d \sim 140 \mathrm{pc}$ Kenyon et al. 2008 and references therein). However, some of our targets were expected to be located very close to the Sun because of their late spectral types and relative brightness. We used the absolute magnitude $M_{J}$-spectral type relation in Caballero et al. (2008) for deriving spectro-photometric distances for the 24 investigated field $\mathrm{M}$ dwarfs, which are given in Table 4. We assumed generous uncertainties in the $M_{J}$-SpT relation, apart from those in our SpT determination and the 2MASS $J$ magnitudes, which translated into typical error bars of about $20 \%$. There have been previous determinations of the distances to three known dwarfs based solely on VI-band (Fleming 1998) and optical and near-infrared photometry (Caballero 2012). Those determinations are consistent with ours within conservative error bars.

All of our field $\mathrm{M}$ dwarfs except four (which are M2.5-3.5 V stars) are located at less $25 \mathrm{pc}$. Of them, seven are at $15 \mathrm{pc}$ or less, of which only three were previously known (J0122+2209/G 34-53 at $8 \pm 2 \mathrm{pc}, \mathrm{J} 0156+3033 / \mathrm{NLTT} 6496$ at $15 \pm 4 \mathrm{pc}$, and $\mathrm{J} 2211+4059 / 1 \mathrm{RXS} \mathrm{J} 221124.3+410000$ at $9 \pm 2 \mathrm{pc}$ ). The other four were identified in this work for the first time (J0012+3028, J0507+3730, J1518+2036, and $\mathrm{J} 2259+3738$ at $12-15 \mathrm{pc})$.

\subsection{Common Proper Motion Pairs}

We took advantage of the Aladin sky atlas and the VOTable Plotting tool VOplot to look for proper-motion companions to our 27 stars. We loaded PPMXL data in a circular area of 30 arcmin radius centered on our targets and plotted proper-motion diagrams $\left(\mu_{\delta}\right.$ versus $\left.\mu_{\alpha} \cos \delta\right)$. We recovered one known binary system (Königstuhl 4 AB; Caballero 2012) and reported and characterized for the first time another one.

The new binary, not tabulated in the Washington Double Star Catalog (Mason et al. 2001), consists of 

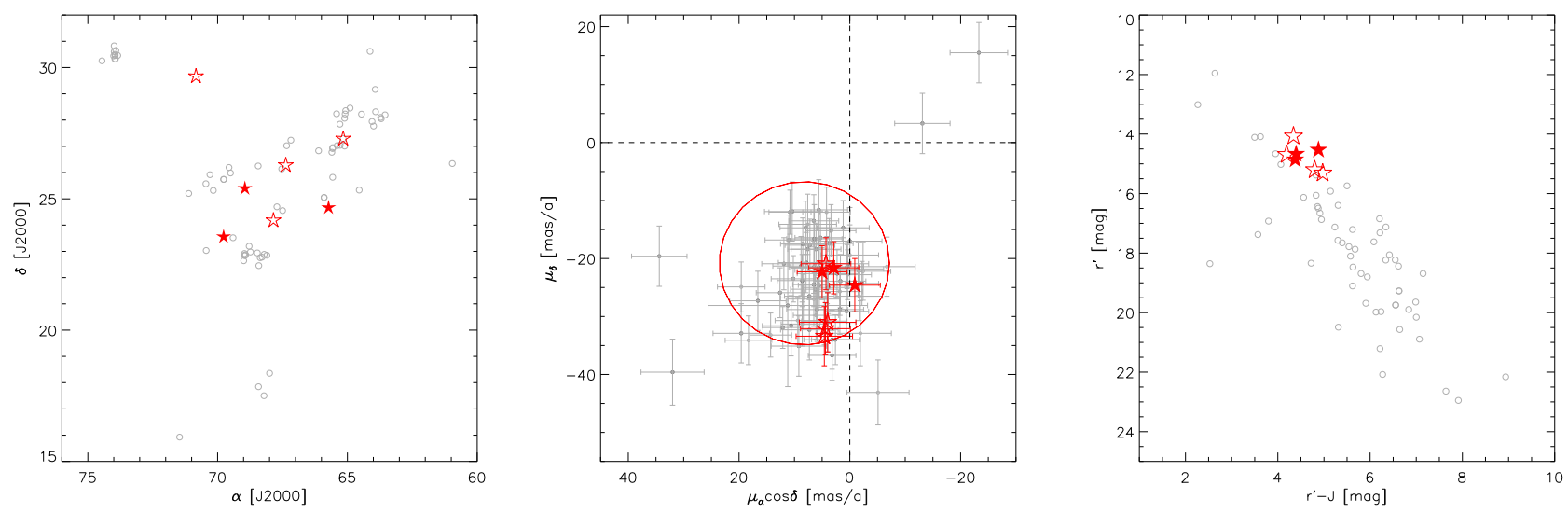

FIG. 6. - Left panel: spatial distribution of candidate members in the Taurus-Auriga star-forming region. (Red) filled stars are our three new candidates with IDS/INT spectroscopy, (red) open stars are the four known Taurus-Auriga T Tauri stars identified in the 2MASSCMC14 cross-match and listed in Table17 and (gray) open circles are Taurus-Auriga members from/Luhman (2004), Luhman et al. (2006, 2009), and Guieu et al. (2006). Middle panel: same as the left panel, but for the proper-motion diagram. The ellipse indicates the average values and standard deviations of proper motions in Taurus-Auriga from Bertout \& Genova (2006), Right panel: same as the left panel, but for the $r^{\prime}$ vs. $r^{\prime}-J$ color-magnitude diagram.

J0326+3929/LSPM J0326+3929E and its close companion LSPM J0326+3929W. The two stars were reported first by Lépine \& Shara (2005), who did not provide any clues of their possible binarity (the Lépine \& Shara 2005 catalog is a very useful source of new proper motion pairs identified by amateur astronomers - e.g., López et al. 2012; Rica 2012). However, because of its angular separation that is shorter than 55 arcsec and identical proper motions, the pair probably is one of the 19,836 highly probable wide binaries reported by Lépine (2011).

We applied the method of (Caballero 2007) of confirming membership in a common proper motion pair by comparing multi-band photometry (in this case, $\left.u^{\prime} g^{\prime} r^{\prime} i^{\prime} J H K_{s} W 1 W 2 W 3\right)$ of the two components and measuring constant angular separation $\rho$ and position angle $\theta$ on a long time baseline. Table 6 summarizes our astrometric analysis of SuperCOSMOS digitizations of the First and Second Palomar Observatory Sky Survey (Hambly et al. 2001) and other public databases. With a significant proper motion of $\mu \sim 160$ mas a $^{-1}$, the two stars would be separated by up to 15 arcsec in the 1955 POSS-I images if the secondary star were in the background. However, $\rho$ and $\theta$ kept constant at $6.3 \pm 0.3$ arcsec and $229 \pm 3 \mathrm{deg}$ in a 55.4 a long interval, from which we concluded that the two stars travel together. Both LSPM J0326+3929E and W were saturated in all POSS photographic plates and, because of their proximity, there were large uncertainties in the determination of the photocentroids. We determined more precise mean angular separation and position angle at $\rho=6.48 \pm 0.16 \operatorname{arcsec}$ and $\theta=$ $227.9 \pm 1.0 \mathrm{deg}$ by averaging only the last four astrometric epochs $(\Delta t=11.7 \mathrm{a})$. With the distance computed in Section $3.5(d=17 \pm 4 \mathrm{pc})$, we derived a projected physical separation of $s=110 \pm 30 \mathrm{au}$. From the spectral type of the primary, M4.5 V, and the magnitude differences between the two components, of $\Delta r^{\prime}=0.357 \pm 0.004 \mathrm{mag}$ and $\Delta J=0.28 \pm 0.04 \mathrm{mag}$, we estimated a spectral type M5.0: $\mathrm{V}$ for the secondary.

\section{DISCUSSION AND CONCLUSIONS}

We showed the potential of the Virtual Observatory for finding new bright nearby $M$ dwarfs, some of which can be targeted by current or forthcoming exoplanet sur- veys. In this pilot program, we cross-matched the photometric CMC14 $\left(r^{\prime}\right)$ and 2MASS $\left(J H K_{s}\right)$ catalogs in the whole overlapping area of $25078 \mathrm{deg}^{2}$, imposed color restrictions in $r^{\prime}-J$ and $J-K_{s}$, and selected 828 sources brighter than $J=10.5 \mathrm{mag}$ for follow-up. Some of them turned out to be background M giants or even reddened, massive early-type stars in distant open clusters. Proper motions were used in a second step in giving priorities in the spectroscopic follow-up.

We used the Intermediate Dispersion Spectrograph at the $2.5 \mathrm{~m}$ Isaac Newton Telescope for obtaining lowresolution optical spectroscopy of 27 targets, 25 of which had not been spectroscopically analyzed before. We determined spectral types with a custom-made spectral index, $\Re$, which accounts mostly for the absorption of a TiO band at $7100-7500 \AA$. Derived spectral types of all the stars ranged between $\mathrm{M} 2.5 \mathrm{~V}$ and $\mathrm{M} 5.5 \mathrm{~V}$, with the bulk of them in the narrower M3.5-5.0 V interval, which demonstrated the success of our search.

In spite of their relative brightness, $J<10.2 \mathrm{mag}$ in seven cases (and $J<9.7 \mathrm{mag}$ in one case), $16(60 \%)$ $\mathrm{M}$ dwarfs had escaped previous surveys and are, therefore, discovered and characterized here for the first time. This fact may be due to that our survey, contrary to most searches for $\mathrm{M}$ dwarfs, was purely photometric and that most of our stars fell above the main-sequence locus in a reduced-proper-motion diagram. That is, proper motions of our targets are lower than average for typical dwarfs of the same $r^{\prime}-J$ colors. Without an appropriate radial-velocity study, one cannot deduce low total Galactic velocity from low tangential velocity ( $M$ dwarfs can move fast in the visual direction instead), but one can at least conclude that proper-motion surveys are inefficient in the identification of slow M dwarfs, even if they are bright and nearby.

Indeed, among our $27 \mathrm{M}$ dwarfs, there are two stars at less than $10 \mathrm{pc}$, to which we recommend measuring the parallax: J0122+2209/G 34-53 (M4.0 V, $d=8 \pm 2 \mathrm{pc})$ and J2211+4059/1RXS J221124.3+410000 (M5.5 V, $d=$ $9 \pm 2 \mathrm{pc})$. There are another five stars at $10-15 \mathrm{pc}$, four of which are presented here for the first time. The identification of new relatively bright, low-active, single stars 
much closer to Earth than the median distance to Mdwarf exoplanet-survey targets $(\gg 13 \mathrm{pc})$ is still a matter of interest. In summary, this kind of VO color-based search may shed light on the complete identification and characterization of all $\mathrm{M}$ dwarfs in the $10 \mathrm{pc}$ radius sphere centered on the Sun, until the ESA space mission Gaia delivers its final catalog by 2022 .

We were genuinely surprised by the discovery of three slow M dwarfs with low surface gravities from weak Na I absorption in our IDS/INT spectra and from VOSA fits to observed multi-wavelength spectral energy distributions. Besides, one of them, J0422+2439, had a strong $\mathrm{H} \alpha$ emission indicative of accretion (the $\mathrm{pEW}(\mathrm{H} \alpha) \mathrm{s}$ of the other 26 stars were consistent with the chromospheric activity). This fact led us to study the X-ray emission of the sample stars in the ROSAT Bright Source Catalogue. Of the 11 (40\%) positive cross-matches with ROSAT, 7 were new detections, which suggests that previous all-sky X-ray surveys for low-mass stars have been incomplete. The coolest star in our sample, J2211+4059 (M5.5 V), had also the highest $L_{X} / L_{J}$ ratio, slightly above those of four other stars, including J0422+2439.

We assigned membership of J0422+2439 to the TaurusAuriga star-forming region based not only on low surface gravity and $\mathrm{H} \alpha$ and X-ray emissions, but also on coincidence of spatial location, proper motions, and colormagnitude combinations with a large sample of known Taurus-Auriga members. We also assigned membership of J0435+2523 and J0439+2333, the other two lowgravity stars, in the star-forming region. The identification of three new intermediate $\mathrm{M}$ dwarfs in TaurusAuriga may help alleviate the reported lack of them, which has made many authors to claim the uniqueness of the initial mass function in Taurus-Auriga.

We also looked for proper-motion companions to our 27 stars. We recovered a fragile, wide, already known pair and reported and characterized a new pair, an $\mathrm{M} 4.5 \mathrm{~V}$ star and an M5.0: V companion separated by 6.5 arcsec $(\sim 110 \mathrm{AU})$.

We will continue to search with VO tools for slow bright nearby $\mathrm{M}$ dwarfs, in particular for potential targets for exoplanet hunting. For that, we will not only plan to conclude the analysis of our CMC14+2MASS data with a new spectroscopic follow-up, but also start a new study with the latest release of the Carlsberg Meridian Catalogue ( $\mathrm{CMC} 158)$, which will be more extensive than the CMC14 one. Extra VO works for the identification of slow bright nearby $\mathrm{M}$ dwarfs unnoticed by previous surveys will include massive cross-matches between existing databases relevant for this topic: 2MASS, PPMXL, UCAC4, WISE, GALEX, ROSAT, VISTA, and VST. These works will pave the way for further 'super-massive' correlations when the first Gaia and $E U C L I D$ data releases are available.

We gratefully thank F. J. Alonso-Floriano, P. CruzGamba, A. Klutsch, and B. Stelzer for their helpful feedback and data provision. This publication is based on observations made with the Isaac Newton Telescope operated on the island of La Palma by the Isaac Newton Group in the Spanish Observatorio del Roque de los Muchachos of the Instituto de Astrofísica de Canarias. This publication has made use of the SIMBAD, VizieR and Aladin, operated at center de Données astronomiques de Strasbourg, France, the Washington Double Star Catalog maintained at the U.S. Naval Observatory, and VOSA, a Virtual Observatory tool developed under the Spanish Virtual Observatory project supported from the Spanish MICINN through grant AyA2008-02156. MCGO acknowledges the support of a JAE-Doc CSIC fellowship cofunded with the European Social Fund under the program Junta para la Ampliación de Estudios. Financial support was provided by the Spanish Ministerio de Ciencia e Innovación under grants AyA2011-24052 and AYA2011-30147-C03-03.

\section{REFERENCES}

Aberasturi, M., Solano, E., \& Martín, E. L. 2011, A\&A, 534, L7 Aihara, H., Allende Prieto, C., An, D., et al. 2011, ApJS, 193, 29 Allard, F. 2014, in IAU Symposium, Vol. 299, IAU Symposium, ed. M. Booth, B. C. Matthews, \& J. R. Graham, 271-272

Anglada-Escudé, G., Arriagada, P., Vogt, S. S., et al. 2012, ApJ, $751, \mathrm{~L} 16$

Apps, K., Clubb, K. I., Fischer, D. A., et al. 2010, PASP, 122, 156

Artigau, É., Donati, J.-F., \& Delfosse, X. 2011, in Astronomical Society of the Pacific Conference Series, Vol. 448, 16th Cambridge Workshop on Cool Stars, Stellar Systems, and the Sun, ed. C. Johns-Krull, M. K. Browning, \& A. A. West, 771

Barrado y Navascués, D., \& Martín, E. L. 2003, AJ, 126, 2997

Batalha, N. M., Borucki, W. J., Koch, D. G., et al. 2010, ApJ, 713, L109

Bayo, A., Rodrigo, C., Barrado Y Navascués, D., et al. 2008, A\&A, 492, 277

Bean, J., Seifahrt, A., Hartman, H., et al. 2010, The Messenger, 140,41

Béjar, V. J. S., Zapatero Osorio, M. R., \& Rebolo, R. 1999, ApJ, 521,671

Berta, Z. K., Irwin, J., \& Charbonneau, D. 2013, ApJ, 775, 91

Berta, Z. K., Irwin, J., Charbonneau, D., Burke, C. J., \& Falco, E. E. 2012, AJ, 144, 145

Bertout, C., \& Genova, F. 2006, A\&A, 460, 499

\footnotetext{
8 http://svo2.cab.inta-csic.es/vocats/cmc15/
}

Blake, C. H., Charbonneau, D., \& White, R. J. 2010, ApJ, 723, 684

Bochanski, J. J., Hawley, S. L., Covey, K. R., et al. 2010, AJ, 139, 2679

Bonfils, X., Gillon, M., Forveille, T., et al. 2011, A\&A, 528, A111

Bonfils, X., Delfosse, X., Udry, S., et al. 2013, A\&A, 549, A109

Bonnarel, F., Fernique, P., Bienaymé, O., et al. 2000, A\&AS, 143, 33

Briceño, C., Hartmann, L., Stauffer, J., \& Martín, E. 1998, AJ, 115,2074

Briceño, C., Luhman, K. L., Hartmann, L., Stauffer, J. R., \& Kirkpatrick, J. D. 2002, ApJ, 580, 317

Browning, M. K., Basri, G., Marcy, G. W., West, A. A., \& Zhang, J. 2010, AJ, 139, 504

Burgasser, A. J., Kirkpatrick, J. D., Brown, M. E., et al. 2002, ApJ, 564, 421

Burningham, B., Naylor, T., Littlefair, S. P., \& Jeffries, R. D. 2005, MNRAS, 356, 1583

Burningham, B., Leggett, S. K., Lucas, P. W., et al. 2010, MNRAS, 404, 1952

Caballero, J. A. 2007, ApJ, 667, 520

Caballero, J. A. 2009, in Multi-wavelength Astronomy and

Virtual Observatory, ed. D. Baines \& P. Osuna, 3

-. 2012, The Observatory, 132, 1

Caballero, J. A., Albacete-Colombo, J. F., \& López-Santiago, J. 2010, A\&A, 521, A45 
Caballero, J. A., Burgasser, A. J., \& Klement, R. 2008, A\&A, 488, 181

Caballero, J. A., Cortés-Contreras, M., Alonso-Floriano, F. J., et al. 2013, in Protostars and Planets VI, Heidelberg, July 15-20, 2013. Poster \#2K020, 20

Charbonneau, D., Berta, Z. K., Irwin, J., et al. 2009, Nature, 462, 891

Chauvin, G., Lagrange, A.-M., Bonavita, M., et al. 2010, A\&A, 509, A52

Comerón, F., Pasquali, A., Rodighiero, G., et al. 2002, A\&A, 389, 874

Copenhagen University, O., Institute, A. O., Cambridge, Uk, \& Real Instituto Y Observatorio de La Armada, F. E. S. 2006, VizieR Online Data Catalog, 1304, 0

Cruz, K. L., \& Reid, I. N. 2002, AJ, 123, 2828

Cushing, M. C., Kirkpatrick, J. D., Gelino, C. R., et al. 2011, ApJ, 743, 50

Cutri, R. M., \& et al. 2012, VizieR Online Data Catalog, 2311, 0

Dhital, S., West, A. A., Stassun, K. G., \& Bochanski, J. J. 2010, AJ, 139, 2566

Evans, D. W., Irwin, M. J., \& Helmer, L. 2002, A\&A, 395, 347

Faherty, J. K., Burgasser, A. J., West, A. A., et al. 2010, AJ, 139, 176

Fleming, T. A. 1998, ApJ, 504, 461

Frith, J., Pinfield, D. J., Jones, H. R. A., et al. 2013, MNRAS, 435,2161

Fuhrmeister, B., \& Schmitt, J. H. M. M. 2003, A\&A, 403, 247

Gershberg, R. E., Katsova, M. M., Lovkaya, M. N., Terebizh, A. V., \& Shakhovskaya, N. I. 1999, A\&AS, 139, 555

Giclas, H. L., Burnham, R., \& Thomas, N. G. 1961, Lowell Observatory Bulletin, 5, 61

- 1971, Lowell proper motion survey Northern Hemisphere.

Giclas, H. L., Burnham, Jr., R., \& Thomas, N. G. 1978, Lowell Observatory Bulletin, 8, 89

Giclas, H. L., Slaughter, C. D., \& Burnham, R. 1959, Lowell Observatory Bulletin, 4, 136

Gizis, J. E., Monet, D. G., Reid, I. N., et al. 2000, AJ, 120, 1085

Gliese, W. 1969, Veroeffentlichungen des Astronomischen Rechen-Instituts Heidelberg, 22, 1

Gliese, W., \& Jahreiss, H. 1991, NASA STI/Recon Technical Report A, 92, 33932

Güdel, M., Briggs, K. R., Arzner, K., et al. 2007, A\&A, 468, 353

Guieu, S., Dougados, C., Monin, J.-L., Magnier, E., \& Martín, E. L. 2006, A\&A, 446, 485

Haakonsen, C. B., \& Rutledge, R. E. 2009, ApJS, 184, 138

Hambly, N. C., MacGillivray, H. T., Read, M. A., et al. 2001, MNRAS, 326, 1279

Hartman, J. D., Bakos, G. Á., Noyes, R. W., et al. 2011, AJ, 141, 166

Hawley, S. L., Gizis, J. E., \& Reid, I. N. 1996, AJ, 112, 2799

Hawley, S. L., Gizis, J. E., \& Reid, N. I. 1997, AJ, 113, 1458

Hawley, S. L., et al. 2002, AJ, 123, 3409

Helou, G., \& Walker, D. W. 1988, Infrared astronomical satellite (IRAS) catalogs and atlases. Volume 7: The small scale structure catalog, 7

Howard, A. W., Marcy, G. W., Bryson, S. T., et al. 2012, ApJS, 201,15

Irwin, J. M., Quinn, S. N., Berta, Z. K., et al. 2011, ApJ, 742, 123

Jiménez-Esteban, F. M., Caballero, J. A., Dorda, R., Miles-Páez, P. A., \& Solano, E. 2012, A\&A, 539, A86

Johnson, J. A., Howard, A. W., Marcy, G. W., et al. 2010, PASP, 122,149

Kenyon, S. J., Gómez, M., \& Whitney, B. A. 2008, Book, 405

Kirkpatrick, J. D., Henry, T. J., \& McCarthy, Jr., D. W. 1991, ApJS, 77, 417

Kirkpatrick, J. D., Reid, I. N., Liebert, J., et al. 1999, ApJ, 519, 802

Konopacky, Q. M., Ghez, A. M., Barman, T. S., et al. 2010, ApJ, 711,1087

Leggett, S. K., Allard, F., Dahn, C., et al. 2000, ApJ, 535, 965

Lépine, S. 2011, Cool Stars in Wide Binaries: 23,000 Common Proper Motion Doubles from the SUPERBLINK Proper Motion Survey, 448, 1375

Lépine, S., \& Gaidos, E. 2011, AJ, 142, 138

Lépine, S., Hilton, E. J., Mann, A. W., et al. 2013, AJ, 145, 102

Lépine, S., Rich, R. M., \& Shara, M. M. 2003a, AJ, 125, 1598
Lépine, S., \& Shara, M. M. 2005, AJ, 129, 1483

Lépine, S., Shara, M. M., \& Rich, R. M. 2003b, AJ, 126, 921

Lodieu, N., Espinoza Contreras, M., Zapatero Osorio, M. R., et al. 2012, A\&A, 542, A105

López, C., Calandra, F., Chalela, M., et al. 2012, Journal of Double Star Observations, 8, 73

López Martí, B., Jiménez-Esteban, F., Bayo, A., et al. 2013, A\&A, 556, A144

Luhman, K. L. 2004, ApJ, 617, 1216

-. 2013, ApJ, 767, L1

Luhman, K. L., Briceño, C., Stauffer, J. R., et al. 2003, ApJ, 590, 348

Luhman, K. L., Mamajek, E. E., Allen, P. R., \& Cruz, K. L. 2009, ApJ, 703, 399

Luhman, K. L., Whitney, B. A., Meade, M. R., et al. 2006, ApJ, 647,1180

Luyten, W. J. 1979a, LHS catalogue. A catalogue of stars with proper motions exceeding 0" 5 annually

- 1979b, New Luyten catalogue of stars with proper motions larger than two tenths of an arcsecond; and first supplement; NLTT. (Minneapolis (1979))

Mahadevan, S., Ramsey, L., Bender, C., et al. 2012, in Society of Photo-Optical Instrumentation Engineers (SPIE) Conference Series, Vol. 8446, Society of Photo-Optical Instrumentation Engineers (SPIE) Conference Series

Malo, L., Doyon, R., Lafrenière, D., et al. 2013, ApJ, 762, 88

Martín, E. L., Basri, G., Delfosse, X., \& Forveille, T. 1997, A\&A, 327, L29

Martín, E. L., Cabrera, J., Martioli, E., Solano, E., \& Tata, R. 2013, A\&A, 555, A108

Martín, E. L., Delfosse, X., Basri, G., et al. 1999, AJ, 118, 2466

Martín, E. L., Dougados, C., Magnier, E., et al. 2001, ApJ, 561, L195

Martín, E. L., Rebolo, R., \& Zapatero-Osorio, M. R. 1996, ApJ, 469,706

Martín, E. L., Phan-Bao, N., Bessell, M., et al. 2010, A\&A, 517, A53

Mason, B. D., Wycoff, G. L., Hartkopf, W. I., Douglass, G. G., \& Worley, C. E. 2001, AJ, 122, 3466

McCarthy, M. F., \& Treanor, P. J. 1964, Ricerche Astronomiche, 6,535

McGovern, M. R., Kirkpatrick, J. D., McLean, I. S., et al. 2004, ApJ, 600, 1020

Miller-Ricci, E., \& Fortney, J. J. 2010, ApJ, 716, L74

Mochnacki, S. W., Gladders, M. D., Thomson, J. R., et al. 2002, AJ, 124, 2868

Mooley, K., Hillenbrand, L., Rebull, L., Padgett, D., \& Knapp, G. 2013, ApJ, 771, 110

Morin, J., Donati, J.-F., Petit, P., et al. 2010, MNRAS, 407, 2269

Muirhead, P. S., Hamren, K., Schlawin, E., et al. 2012, ApJ, 750, L37

Mundt, R., Alonso-Floriano, F. J., Caballero, J. A., et al. 2013, in Protostars and Planets VI, Heidelberg, July 15-20, 2013. Poster \#2K055, 55

Muzerolle, J., Hillenbrand, L., Calvet, N., Briceño, C., \& Hartmann, L. 2003, ApJ, 592, 266

Perger, M., Lodieu, N., Martín, E. L., \& Barrado, D. 2013, Mem. Soc. Astron. Italiana, 84, 948

Quirrenbach, A., Amado, P. J., Seifert, W., et al. 2012, in Society of Photo-Optical Instrumentation Engineers (SPIE) Conference Series, Vol. 8446, Society of Photo-Optical Instrumentation Engineers (SPIE) Conference Series

Rajpurohit, A. S., Reylé, C., Allard, F., et al. 2013, A\&A, 556, A15

Reid, I. N., Hawley, S. L., \& Gizis, J. E. 1995, AJ, 110, 1838

Reid, I. N., Cruz, K. L., Allen, P., et al. 2003, AJ, 126, 3007

Reiners, A., Bean, J. L., Huber, K. F., et al. 2010, ApJ, 710, 432

Rica. 2012, Journal of Double Star Observations, 8, 73

Ricker, G. R., Latham, D. W., Vanderspek, R. K., et al. 2010, in Bulletin of the American Astronomical Society, Vol. 42, American Astronomical Society Meeting Abstracts \#215, 450.06

Roeser, S., Demleitner, M., \& Schilbach, E. 2010, AJ, 139, 2440

Rojas-Ayala, B., Covey, K. R., Muirhead, P. S., \& Lloyd, J. P.

2010, ApJ, 720, L113

-. 2012, ApJ, 748, 93

Ross, F. E. 1939, AJ, 48, 163 
Scelsi, L., Maggio, A., Micela, G., et al. 2007, A\&A, 468, 405 Schlaufman, K. C., \& Laughlin, G. 2010, A\&A, 519, A105

Schlieder, J. E., Lépine, S., Rice, E., et al. 2012, AJ, 143, 114

Schmitt, J. H. M. M., Fleming, T. A., \& Giampapa, M. S. 1995, ApJ, 450, 392

Seeliger, M., Neuhäuser, R., \& Eisenbeiss, T. 2011, Astronomische Nachrichten, 332, 821

Shkolnik, E. L., Liu, M. C., Reid, I. N., Dupuy, T., \& Weinberger, A. J. 2011, ApJ, 727, 6

Skrutskie, M. F., et al. 2006, AJ, 131, 1163

Slesnick, C. L., Carpenter, J. M., \& Hillenbrand, L. A. 2006a, AJ, 131,3016

Slesnick, C. L., Carpenter, J. M., Hillenbrand, L. A., \& Mamajek, E. E. 2006b, AJ, 132, 2665

Soderblom, D. R., Hillenbrand, L. A., Jeffries, R. D., Mamajek, E. E., \& Naylor, T. 2013, ArXiv e-prints, arXiv:1311.7024

Steele, I. A., \& Jameson, R. F. 1995, MNRAS, 272, 630

Stelzer, B., Marino, A., Micela, G., López-Santiago, J., \& Liefke, C. 2013, MNRAS, 431, 2063

Taylor, M. B. 2006, in Astronomical Society of the Pacific Conference Series, Vol. 351, Astronomical Data Analysis Software and Systems XV, ed. C. Gabriel, C. Arviset, D. Ponz, \& S. Enrique, 666
Tinetti, G., Beaulieu, J. P., Henning, T., et al. 2012, Experimental Astronomy, 34, 311

Valdivielso, L., Martín, E. L., Bouy, H., et al. 2009, A\&A, 497, 973

van Maanen, A. 1915, ApJ, 41, 187

Voges, W., Aschenbach, B., Boller, T., et al. 1999, A\&A, 349, 389

West, A. A., Hawley, S. L., Bochanski, J. J., et al. 2008, AJ, 135, 785

West, A. A., Hawley, S. L., Walkowicz, L. M., et al. 2004, AJ, 128,426

West, A. A., Morgan, D. P., Bochanski, J. J., et al. 2011, AJ, 141, 97

Wolf, M. 1919, Veroeffentlichungen der Badischen Sternwarte zu Heidelberg, 7, 195

Zacharias, N., Finch, C. T., Girard, T. M., et al. 2012, VizieR Online Data Catalog, 1322, 0

Zasowski, G., Johnson, J. A., Frinchaboy, P. M., et al. 2013, AJ, 146,81

Zickgraf, F.-J., Engels, D., Hagen, H.-J., Reimers, D., \& Voges, W. 2003, A\&A, 406, 535 